\title{
Gravity currents from a dam-break in a rotating channel
}

\author{
By KARL R. HELFRICH AND JULIA C. MULLARNEY ${ }^{1} \dagger$ \\ ${ }^{1}$ Department of Physical Oceanography, Woods Hole Oceanographic Institution, \\ Woods Hole, MA 02543, USA \\ ${ }^{2}$ Research School of Earth Sciences, Australian National University, Canberra, Australia
}

(Received 4 September 2004 and in revised form 10 February 2005)

The generation of a gravity current by the release of a semi-infinite region of buoyant fluid of depth $H$ overlying a deeper, denser and quiescent lower layer in a rotating channel of width $w$ is considered. Previous studies have focused on the characteristics of the gravity current head region and produced relations for the gravity current speed $c_{b}$ and width $w_{b}$ as a functions of the local current depth along the wall $h_{b}$, reduced gravity $g^{\prime}$, and Coriolis frequency $f$. Here, the dam-break problem is solved analytically by the method of characteristics assuming reducedgravity flow, uniform potential vorticity and a semigeostrophic balance. The solution makes use of a local gravity current speed relation $c_{b}=c_{b}\left(h_{b}, \ldots\right)$ and a continuity constraint at the head to close the problem. The initial value solution links the local gravity current properties to the initiating dam-break conditions. The flow downstream of the dam consists of a rarefaction joined to a uniform gravity current with width $w_{b}(\leqslant w)$ and depth on the right-hand wall of $h_{b}$, terminated at the head moving at speed $c_{b}$. The solution gives $h_{b}, c_{b}, w_{b}$ and the transport of the boundary current as functions of $w / L_{R}$, where $L_{R}=\sqrt{g^{\prime} H} / f$ is the deformation radius. The semigeostrophic solution compares favourably with numerical solutions of a singlelayer shallow-water model that internally develops a leading bore. Existing laboratory experiments are re-analysed and some new experiments are undertaken. Comparisons are also made with a three-dimensional shallow-water model. These show that lateral boundary friction is the primary reason for differences between the experiments and the semigeostrophic theory. The wall no-slip condition is identified as the primary cause of the experimentally observed decrease in gravity current speed with time. A model for the viscous decay is developed and shown to agree with both experimental and numerical model data.

\section{Introduction}

Rotating gravity currents are important features of numerous geophysical flows (Griffiths 1986; Simpson 1997). Examples include the penetration of coastally trapped disturbances in the marine atmospheric boundary layer (Beardsley et al. 1987; Dorman 1987) and the discharge of relatively fresh river water into the coastal ocean (e.g. Munchow \& Garvine 1993; Rennie, Largier \& Lentz 1999). Studies of rotating gravity currents have typically focused on the local dynamics of the gravity current head produced by a dam-break in a rectangular channel. The rupture of the dam

$\dagger$ Present address: Department of Oceanography, Dalhousie University, Halifax N.S., Canada. 
leads to a narrow gravity current that propagates along the right-hand wall of the channel (looking downstream with northern hemisphere rotation). The experiments showed an approximately laminar geostrophic boundary current that terminates at a generally turbulent blunt bore-like nose from which fluid approaching the head from behind may be detrained (Stern, Whitehead \& Hua 1982; Griffiths \& Hopfinger 1983; Kubokawa \& Hanawa 1984). The bore speed $c_{b}=\beta \sqrt{g^{\prime} h_{b}}$, where $h_{b}$ is the depth of the boundary current at the wall immediately upstream of the turbulent head and $g^{\prime}=g \Delta \rho / \rho_{2}$ is the reduced gravity, $\Delta \rho$ is the density difference between the ambient $\left(\rho_{2}\right)$ and gravity current fluids. These three studies found $\beta \approx 1.1,1.3$ and 1.0 , respectively. The speed can be related to the maximum depth of the turbulent bore which is about $1.3 h_{b}$ (Griffiths \& Hopfinger 1983).

An important aspect of the Stern et al. (1982) (hereinafter referred to as SWH) and Griffiths \& Hopfinger (1983) (hereinafter referred to as GH) experiments was that in many runs, $c_{b}$ decreased with time. SWH found that in some cases, the bore stagnated and formed a growing gyre at the end of the gravity current. GH attributed the speed decay to drag from Taylor column formation and radiation of inertial waves excited by Kelvin-Helmholtz billows near the bore head.

While the speed of the bore is unambiguous, the definition of gravity current width $w_{b}$ is less clear cut. Turbulence and detrainment at the head lead to multiple possibilities. Additionally, the trailing gravity current may taper toward the front and baroclinic eddies may be present, though GH argue that the eddies are the result of Kelvin-Helmholtz instability occurring in the head region. SWH define the width to be that part of the geostrophic boundary current with velocity in the direction of propagation $u>c_{b}$ (the definition does includes fluid in a viscous wall boundary layer with $u<c_{b}$ ) and excludes the fluid detrained from the bore head. They also averaged the width over some length of the current upstream of the head. GH take $w_{b}$ to be the maximum width of the gravity current head including any detrained fluid. Kubokawa \& Hanawa (1984) define $w_{b}$ as the average total current width in the region immediately behind the bore head. Despite these different in definitions, all three studies found $w_{b}=\beta_{w} \sqrt{g^{\prime} h_{b}} / f$, with constant $\beta_{w} \approx 0.5,0.7$ and 0.8 , respectively.

SWH developed a reduced-gravity shallow-water theory for the gravity current. Two self-similar solutions of the long-wave, or semigeostrophic, equations describe the shape of the current: a thinning 'wedge' solution and a 'bore-like' solution. Each solution is associated with a Riemann invariant of the system that is uniform for all fluid parcels. The bore-like solution had the property that the front steepened with time and predicted a limiting bore with the maximal upstream gravity current width $\approx 0.42 \sqrt{g^{\prime} h_{b}} / f$. Intrusions initiated in channels wider than this maximum were expected to adjust so that limiting width current propagates downstream. SWH favoured this solution over the wedge because of its bore-like character. They derived a rotating extension to the Bernoulli-conserving, non-rotating bore condition of Benjamin (1968). When joined to the limiting gravity current width, the condition gave $c_{b}=1.57 \sqrt{g^{\prime} h_{b}}$. Imposition of continuity at the bore head implied a detrainment of $32 \%$ of the gravity current flux at the bore head.

Kubokawa \& Hanawa (1984) discussed solutions to the same semigeostrophic equations as SWH. Instead of assuming a bore with the SWH limiting gravity current width, they developed a shock joining theory to arrive at a bore speed condition $c_{b} \approx 1.45 \sqrt{g^{\prime} h_{b}}$ and width $w_{b} \approx 0.68 \sqrt{g^{\prime} h_{b}} / f$. No detrainment was predicted since mass continuity was imposed in the shock theory. They argued, but did not demonstrate, that SWH's limiting bore solution would not be realized since a shock could propagate upstream and alter the upstream width. 
Hacker \& Linden (2002) extended an earlier theory by Nof (1987) for steady one and two active layer rotating gravity currents. The analysis assumes energy and momentum conservation, and zero front-relative flow in the gravity current. For a single active layer case of interest here, they found $\beta=2^{1 / 2}$ with $\beta_{w}=2^{-1 / 2}$ following from geostrophy. Martin \& Lane-Serff (2005) developed a model with energy loss (in the ambient layer) and recovered Hacker \& Linden's results in the limit of an infinitely deep ambient layer.

None of the rotating gravity relations above are complete solutions to the dambreak problem. They are local conditions for $c_{b}$ and $w_{b}$, and not relations between the gravity current and the reservoir width $w$ and initial depth $H$. Furthermore, the (uniform) Riemann invariant leading to the SWH bore-like solution is inconsistent with an initially stagnant upstream reservoir. This has been demonstrated by Helfrich, Kuo \& Pratt (1999, hereinafter referred to as HKP) with their analytical solution to the single-layer dam-break, or Rossby adjustment, problem in a rotating channel. Rather, it is the rarefaction, or thinning wedge, solution that is consistent with the upstream reservoir. HKP found that at the leading edge, the nose has vanishing width and depth and travels at a constant speed $\geqslant 2\left(g^{\prime} H\right)^{1 / 2}$ that increases with $w$ (i.e. rotation). The solution is the rotating analogue of the classic non-rotating dambreak solution in which the nose of the rarefaction travels down the channel with speed $2\left(g^{\prime} H\right)^{1 / 2}$ (Stoker 1957).

However, as the experimental studies demonstrate, the single-layer solutions with a rarefying nose are not realized in two-layer systems with small $g^{\prime}$, even if the lower layer is infinitely deep. In the non-rotating case, the participation of the lower layer in the momentum and energy budgets of the bore gives $c_{b} \leqslant\left(2 g^{\prime} H\right)^{1 / 2}$ (Benjamin 1968; Klemp, Rotunno \& Skamrock 1994, 1997). With rotation, similar dynamical considerations apply and lead to the theoretical bore conditions given above.

To resolve this problem, it is necessary to modify the HKP solution in such a way that the local two-layer dynamics leading to the gravity current head are included. This is done by matching to the rarefaction solution a local bore condition of the form $c_{b}=c_{b}\left(h_{b}, \ldots\right)$. This approach was first employed for the non-rotating problem by Abbott (1961) and later by Garvine (1981). The result is a theory that links in a dynamically consistent manner the gravity current properties $c_{b}, w_{b}$ and $h_{b}$ to the reservoir conditions $w, H, g^{\prime}$ and $f$. The solution gives the spatial and temporal evolution of the flow outside the ageostrophic and non-hydrostatic bore head, which is simply the downstream terminus of the gravity current. Since the solution is built around the reduced-gravity model, it is restricted to the limit of $H / H_{T} \ll 1$, where $H_{T}$ is the total depth of both layers.

The HKP theory is briefly reviewed and then extended to incorporate a bore condition in $\S 2$. The theory is then favourably compared in $\S 3$ with numerical solutions of the single-layer shallow-water equations in advective form that internally develop a blunt bore. In $\S 4$, the results of some new laboratory experiments and those from SWH are compared with the theory. Some substantial differences with the theory are found and these are explored in $\S 5$ through numerical solutions of a three-dimensional shallow-water model that accounts for aspects of the experiments that are beyond the single-layer model and theory. The numerical solutions agree well with the theory if the sidewalls boundary conditions are stress-free (slip). They are in much better agreement with the experiments if the wall boundary conditions are no-slip. In particular, significant bore speed decay only occurs with the no-slip boundary conditions. A simple theory for the effects of lateral wall friction on bore propagation is developed in $\S 6$ and compared to the numerical solutions and experiments. The results are discussed in $\S 7$. 


\section{Semigeostrophic theory}

Much of the theoretical development follows closely HKP, where readers are referred for greater detail. The basic aspects are summarized and new developments related to the gravity current head are introduced.

Consider a rectangular channel with constant width $w$ rotating about the $z$-axis with Coriolis frequency $f$. The $x$-axis is directed along the channel and the channel walls are at $y= \pm w / 2$. Initially, a buoyant layer of motionless fluid of density $\rho_{1}=\rho_{2}-\Delta \rho$ and depth $H$ fills the channel behind the dam $(x \leqslant 0)$. The buoyant layer rests on an infinitely deep motionless layer of fluid with density $\rho_{2}$. The motion of the upper layer is taken to be governed by the single-layer (reduced-gravity) shallow-water equations (in dimensionless form)

$$
\begin{gathered}
\frac{\partial u}{\partial t}+u \frac{\partial u}{\partial x}+v \frac{\partial u}{\partial y}-v=-\frac{\partial h}{\partial x}, \\
\delta^{2}\left(\frac{\partial v}{\partial t}+u \frac{\partial v}{\partial x}+v \frac{\partial v}{\partial y}\right)+u=-\frac{\partial h}{\partial y}, \\
\frac{\partial h}{\partial t}+\frac{\partial}{\partial x}(u h)+\frac{\partial}{\partial y}(v h)=0 .
\end{gathered}
$$

Here, $x$ has been non-dimensionalized by the along-channel length scale $L, y$ by the deformation radius $L_{R}=\sqrt{g^{\prime} H} / f$, and time $t$ by $L / \sqrt{g^{\prime} H}$. The layer depth $h$ is scaled by $H$, the along-channel velocity $u$ by $\sqrt{g^{\prime} H}$, and the cross-channel velocity $v$ by $\delta \sqrt{g^{\prime} H}$. Here, $g^{\prime}=g \Delta \rho / \rho_{2}$ is the reduced gravity. The parameter $\delta=L_{R} / L$.

It is assumed that $\delta \rightarrow 0$ in (2.1)-(2.3). The resulting semigeostrophic, or longwave, equations are formally not appropriate in the times immediately following the dam-break, especially for wide channels, but do become increasingly valid as the flow propagates down the channel and the length scale $L$ increases (HKP; Stern \& Helfrich 2002).

In this semigeostrophic limit, the potential vorticity

$$
q=\frac{1-\partial u / \partial y}{h}
$$

is conserved following fluid parcels. Combination of (2.4) with the geostrophic balance remaining in (2.2) after taking $\delta=0$ gives

$$
\frac{\partial^{2} h}{\partial y^{2}}-q h=-1
$$

The potential vorticity is scaled with $f / H$. For the motionless initial conditions considered, $q=1$.

After removal of the dam at $t=0$, the layer downstream of the dam may either occupy the whole channel width $w$ (non-dimensionalized by $L_{R}$ ), or may separate from the left-hand wall (looking in the downstream direction). In the latter case, the layer will have a width $w_{e}(x, t)$ and occupy $-w / 2<y<w_{e}-w / 2$. In either situation, the solution to (2.5) with $q=1$ can be written as (Gill 1977)

$$
h(x, y, t)=1-\hat{h}(x, t) \frac{\sinh \left(y-y_{c}(x, t)\right)}{\sinh \left(\frac{1}{2} w_{e}(x, t)\right)}+(\bar{h}(x, t)-1) \frac{\cosh \left(y-y_{c}(x, t)\right)}{\cosh \left(\frac{1}{2} w_{e}(x, t)\right)} .
$$

The along-channel velocity

$$
u(x, y, t)=\hat{h}(x, t) \frac{\cosh \left(y-y_{c}(x, t)\right)}{\sinh \left(\frac{1}{2} w_{e}(x, t)\right)}-(\bar{h}(x, t)-1) \frac{\sinh \left(y-y_{c}(x, t)\right)}{\cosh \left(\frac{1}{2} w_{e}(x, t)\right)},
$$


follows from geostrophy. Here, $y_{c}=\left(w_{e}-w\right) / 2$ is the cross-channel mid-point of the layer and

$$
\begin{aligned}
& \bar{h}=\frac{1}{2}(h(x,-w / 2, t)+h(x, w / 2, t)), \\
& \hat{h}=\frac{1}{2}(h(x,-w / 2, t)-h(x, w / 2, t)) .
\end{aligned}
$$

When the flow is attached to both walls, $w_{e}=w$ and $y_{c}=0$ in (2.6) and (2.7). The dependent variables are $\bar{h}$ and $\hat{h}$. For separated flow, $h(w / 2, y, t)=0$ and $\hat{h}=\bar{h}$. The dependent variables are then $\bar{h}$ and $w_{e}$.

By taking the sum and difference of the along-channel momentum equation (2.1) evaluated along each wall, or the right-hand wall and the free edge of the flow $y=w_{e}-$ $w / 2$ for separated flow, the governing equations reduce to a $2 \times 2$ quasi-linear system (Pratt 1983; HKP)

For attached flow

$$
\frac{\partial \boldsymbol{v}}{\partial t}+\boldsymbol{A} \frac{\partial \boldsymbol{v}}{\partial x}=0
$$

$$
\boldsymbol{v}=\left(\begin{array}{l}
\bar{h} \\
\hat{h}
\end{array}\right)
$$

and

$$
\boldsymbol{A}=\left(\begin{array}{cc}
\hat{h} T^{-1} & \bar{h} T^{-1} \\
T^{3}(\bar{h}-1)+T & \hat{h} T^{-1}
\end{array}\right),
$$

with $T=\tanh (w / 2)$. The characteristics are

$$
\frac{\mathrm{d} x}{\mathrm{~d} t}=c_{ \pm}^{\text {attach }}=\hat{h} T^{-1} \pm \bar{h}^{1 / 2}\left[1-(1-\bar{h}) T^{2}\right]^{1 / 2} .
$$

When the flow is separated

$$
\begin{gathered}
\boldsymbol{v}=\left(\begin{array}{c}
\bar{h} \\
T_{e}
\end{array}\right) \\
\boldsymbol{A}=\left(\begin{array}{cc}
\frac{3 \bar{h}+T_{e}^{2}+T_{e}^{4}(\bar{h}-1)}{2 T_{e}} & \frac{T_{e}^{4}(\bar{h}-1)-\bar{h}^{2}}{2 T_{e}^{2}} \\
\frac{\left(T_{e}^{2}-1\right)^{2}\left[\bar{h}-(1-\bar{h}) T_{e}^{2}\right]}{2\left[\bar{h}+(1-\bar{h}) T_{e}^{2}\right]} & \frac{\left(1-T_{e}^{2}\right)\left[\bar{h}-(1-\bar{h}) T_{e}^{2}\right]}{2 T_{e}}
\end{array}\right),
\end{gathered}
$$

and

$$
\frac{\mathrm{d} x}{\mathrm{~d} t}=c_{ \pm}^{\text {sep }}=\bar{h} T_{e}^{-1} \pm \bar{h}^{1 / 2}\left[1-(1-\bar{h}) T_{e}^{2}\right]^{1 / 2},
$$

with $T_{e}=\tanh \left(w_{e} / 2\right)$.

The Riemann invariant $R_{ \pm}$on each characteristic can be obtained by integration of

$$
\left.\frac{\mathrm{d} v_{2}}{\mathrm{~d} v_{1}}\right|_{ \pm}=\frac{a_{21}}{a_{11}-c_{ \pm}},
$$

where $v_{i}$ and $a_{i j}$ are the elements of $\boldsymbol{v}$ and $\boldsymbol{A}$ in (2.10), and $c_{ \pm}$are the characteristics (Whitham 1974) . In the case of attached flow (2.17) can be integrated to give

$$
R_{ \pm}=c_{ \pm}^{\text {attach }} \pm\left(T^{-1}-T\right) \log \left(2 \bar{h}^{1 / 2} T+2\left[1-(1-\bar{h}) T^{2}\right]^{1 / 2}\right),
$$

while for separated flow, (2.17) must be integrated numerically.

As discussed in HKP, the initial conditions of a motionless uniform-depth layer for $x<0$ imply that one of the Riemann invariants must be the same for all fluid 
parcels. Taking $R_{-}$uniform gives the physically unrealistic result that fluid flows back upstream into the reservoir immediately after removal of the dam. Thus, $R_{+}$must be uniform, and this provides a relation between the two dependent variables (either $\hat{h}$ and $\bar{h}$ for attached flow, or $\bar{h}$ and $T_{e}$ for separated flow) everywhere throughout the flow. The $R_{-}$invariant provides another relation between the dependent variables which can only be satisfied if the dependent variables are constant along each $c_{-}$ characteristic. As a consequence, each $c_{-}$characteristic speed is constant.

Obtaining the rarefaction solution for a given $T$ involves first determining from (2.18) the relation between $\bar{h}$ and $\hat{h}$. The constant $R_{+}$is found by evaluating (2.18) with the initial conditions $\bar{h}=1$ and $\hat{h}=0$. The region of attached flow extends from the location $x_{u p}$ of the signal that propagates upstream into the undisturbed layer to the point $x_{\text {sep }}$ downstream of the dam where the flow separates from the left-hand wall of the channel (figure $1 a$ ). The speed $c_{u p}$ is found from (2.13) with $\bar{h}=1$ and $\hat{h}=0$ to be $\mathrm{d} x / \mathrm{d} t=x_{u p} / t=-1$, the speed of a linear Kelvin wave propagating upstream along the $y=w / 2$ wall. The value of $\bar{h}$ at $x_{\text {sep }}, \bar{h}_{\text {sep }}$, is found by numerical solution of (2.18) with $\hat{h}=\bar{h}$. The speed $c_{\text {sep }}=x_{\text {sep }} / t$ is then determined from $c_{-}^{\text {attach }},(2.13)$. On the $c_{-}$characteristics in the range $c_{u p}<c_{-}<c_{\text {sep }}$, the solution for $\bar{h}$ and $\hat{h}$ is found by simultaneous solution of the attached $R_{+}$and $c_{-}$relations.

The flow is separated from the left-hand wall for $x>x_{\text {sep }}$. The solution in this region is obtained by numerical integration of the ordinary differential equation (2.17) associated with the $R_{+}$invariant with the initial conditions $\bar{h}=\bar{h}_{\text {sep }}$ and $T_{e}=T$. The constant speed of each solution pair, $\bar{h}$ and $T_{e}$, is then found from $c_{-}^{\text {sep }}$ (2.16). The rarefaction solution terminates at a point on the right-hand wall, $x_{\text {nose }}$, where the layer width and depth simultaneously vanish. This point propagates at speed $c_{\text {nose }}$. In the limit $w \rightarrow 0, c_{\text {nose }}=c_{\text {sep }}=2$ and the classic non-rotating dam-break solution is recovered (Stoker 1957). As the channel width is increased, $c_{\text {nose }}$ grows monotonically to 3.80 at $w \rightarrow \infty$, while $c_{\text {sep }}$ decreases to 0 . A schematic of the characteristic curves is given in figure $1(b)$.

\subsection{Gravity current conditions}

Before preceeding to the solution of the full gravity current problem, it is necessary to specify the bore conditions. The theoretical bore conditions discussed in $\S 1$ are all related and easily derived from the semigeostrophic equations as follows. In the present notation the difference of the along-channel momentum equation (2.1) evaluated along each wall, or the right-hand wall and the free edge of the flow $y=w_{e}-w / 2$ for separated flow, gives the continuity equation (Pratt 1983)

$$
\frac{\partial A}{\partial t}+\frac{\partial Q}{\partial x}=0
$$

The momentum equation along the right-hand wall $(y=-w / 2)$ where the $v=0$ is

$$
\frac{\partial u_{R}}{\partial t}+\frac{\partial}{\partial x}\left(\frac{1}{2} u_{R}^{2}+h_{R}\right)=0 .
$$

Here, $u_{R}$ and $h_{R}$ are the velocity and depth on the right-hand wall. From (2.6), and (2.7), the cross-sectional area of the gravity current

$$
A=\int_{-w / 2}^{w_{e}-w / 2} h \mathrm{~d} y=w_{e}+2 T_{e}(\bar{h}-1),
$$


(a)

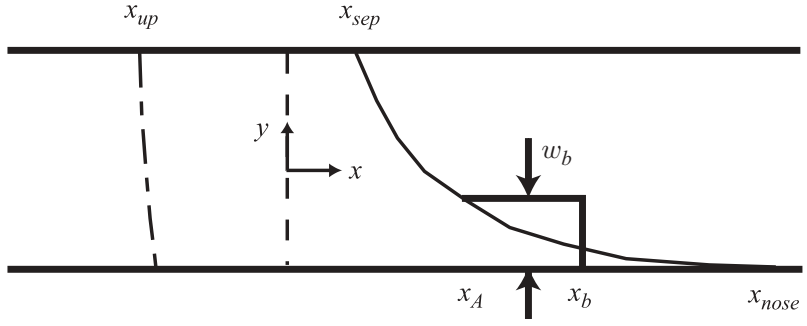

(b)

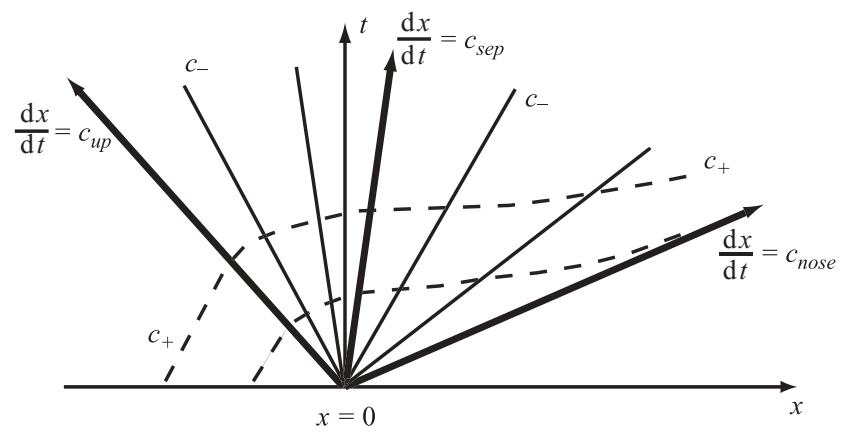

(c)

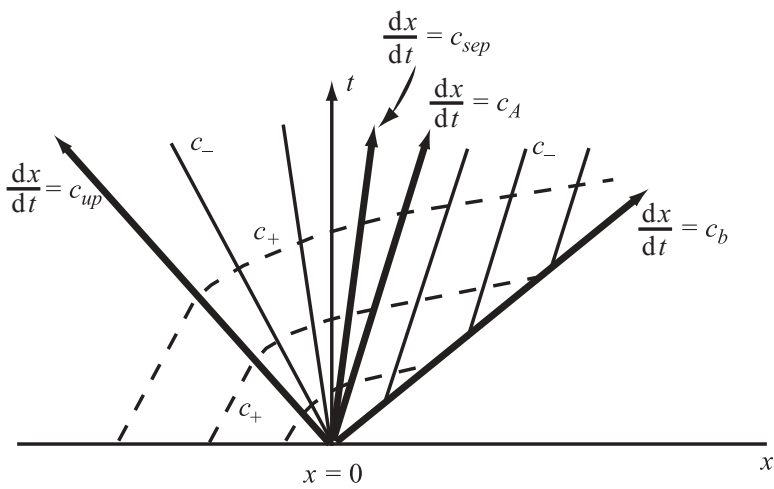

FIGURE 1. (a) Sketch of the surface plan view of a dam-break flow at some time after removal of the dam (the dashed line). The upstream propagating wave is at $x_{u p}$. The downstream rarefaction solution (thin solid line) separates from the left-hand wall at $x_{\text {sep }}$ and terminates as a thin wedge at $x_{\text {nose }}$. The gravity current solution (thick solid line) joins the rarefaction at $x_{A}$ and terminates at the bore head at $x_{b}$ and has width $w_{b}$. (b) Schematic of characteristic curves for the rarefaction solution in HKP. The thin solid (dashed) lines are $c_{-}\left(c_{+}\right)$and characteristics. (c) Same as $(b)$ for a separated gravity current solution.

and the transport

$$
Q=\int_{-w / 2}^{w_{e}-w / 2} u h \mathrm{~d} y=2 \bar{h} \hat{h} .
$$

Recall that if the flow is attached, $w_{e}=w$, whereas if the flow is detached, $\hat{h}=\bar{h}$.

Assuming that the gravity current head propagates steadily at speed $c_{b}$, a RankineHugoniot shock solution to (2.19) and (2.20) can be found. Taking $A, Q, u_{R}$ and $h_{R}=0$ ahead of the gravity current and values behind the bore head indicated by the 
subscript $b$, this solution is

$$
\begin{gathered}
Q_{b}=A_{b} c_{b}, \\
c_{b}=\frac{1}{2} u_{b}+\frac{h_{b}}{u_{b}} .
\end{gathered}
$$

Here, $u_{b}$ is found from (2.7) with $y=-w / 2$ and $h_{b}=\bar{h}+\hat{h}$ for attached flow and $h_{b}=$ $2 \bar{h}$ for separated flow. Equations (2.23) and (2.24) are equivalent to those obtained by Kubokawa \& Hanawa (1984) and (2.24) was derived by SWH. If $u_{b}=\gamma c_{b}$, with $\gamma>0$, (2.24) gives $\beta=c_{b} / h_{b}^{1 / 2}=\left(\gamma-\gamma^{2} / 2\right)^{-1 / 2}$. Thus $\beta \geqslant 2^{1 / 2}$, with the lower bound obtained for $\gamma=1$ as is the case in Benjamin's non-rotating solution and by Hacker \& Linden (2002) with rotation.

The gravity current given by the solution of (2.23) and (2.24) conserves energy in the active layer. It is consistent with the dissipative analyses of Benjamin (1968) and Klemp et al. (1997) who found that the head loss in the lower layer goes to zero as the lower layer becomes infinitely deep. The gravity current becomes energy conserving. The same result occurs in the rotating case (cf. Hacker \& Linden (2002) and Martin \& Lane-Serff (2005) for uniform flow in the active layer).

An alternative to (2.24) is the empirical relation from the laboratory experiments (SWH; GH; Kubokawa \& Hanawa 1984), which in the present non-dimensionalization is

$$
c_{b}=\beta h_{b}^{1 / 2},
$$

where $\beta(\approx 1.2)$ is a constant. Thus, $(2.23)$ (with (2.21)) and either (2.24) or (2.25), give a relation between $\bar{h}$ and either $\hat{h}$ or $T_{e}$ that must be satisfied at the bore.

The continuity condition (2.23) is a bulk kinematic statement and ignores details of the ageostrophic flow in the neighbourhood of the bore head. It leads to a definition of the feeder gravity current that includes all the buoyant fluid within the gravity current, not just fluid with $u>c_{b}$. It does not permit determination of any detrainment, but detainment can be included by multiplying the left-hand side of $(2.23)$ by $\left(1-\delta_{D}\right)$, where $\delta_{D}$ is the specified fraction of the boundary current flux that is detrained at the bore head. In what follows, $\delta_{D}$ will be set to zero.

It is informative to solve the bore conditions (2.23) and (2.24) for $c_{b}$ and $w_{e}$ given $h_{b}$. Figure 2 shows that $c_{b}$ is insensitive to whether the flow is attached or detached (i.e. rotation) $h_{b} \leqslant 1$. Over this range, $\beta=c_{b} / h_{b}^{1 / 2} \approx 1.42$ is nearly constant and only slightly greater than the bound $2^{1 / 2}$. When the flow is detached, $\beta_{w}=w_{e} / h_{b}^{1 / 2} \approx 0.67$ is also nearly constant. These values are slightly smaller than those given in Kubokawa \& Hanawa (1984) for $h_{b} \leqslant 0.5$. It may be that their numerical solution technique introduced a minor error. The slower bore speed from (2.24) with $\beta=1.2$ results in a slightly wider separated gravity current, $\beta_{w} \approx 0.78$.

In the full dam-break problem the depth on the right-hand wall $h_{b} \leqslant 1$, thus when channel width $w \geqslant 0.67$ [0.78] for $c_{b}$ from (2.24) [(2.25)] the gravity current will be separated from the left-hand wall. However, this is an upper bound on the channel width for separated currents. It remains to determine $h_{b}$ from the dam-break initial conditions.

\subsection{Semigeostrophic dam-break gravity current solutions}

The connection of the rarefaction solutions to a bore condition is analogous to the non-rotating dam-break problem in which a piston recedes in the downstream direction at a fixed speed $c_{p}(<2)$ (Stoker 1957). Since the speed of the piston is equal to the local fluid velocity $u$, the layer depth at the piston $h_{b}$ can be found immediately 


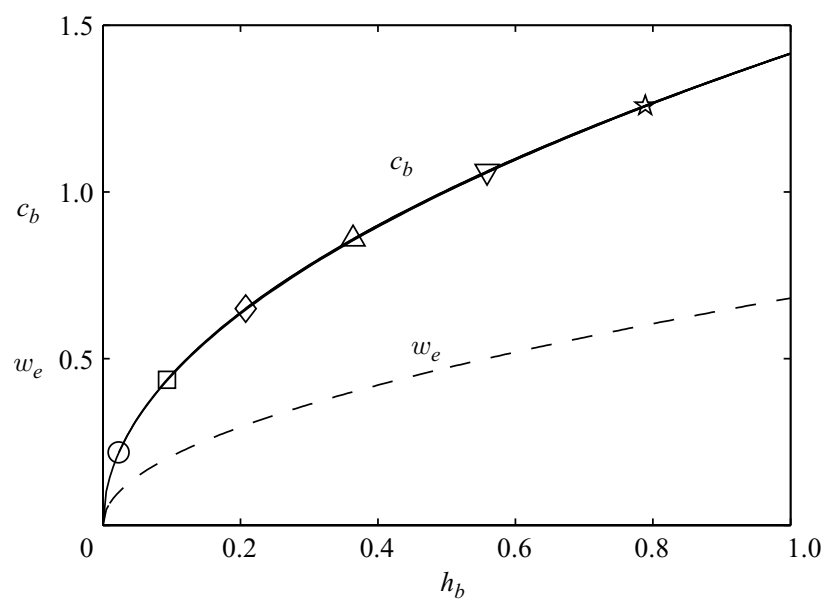

FiguRE 2. The gravity current speed $c_{b}$ (solid) and width $w_{b}$ (dashed) as functions of bore height $h_{b}$ from the semigeostrophic shock solution (2.23) and (2.24). The speed and width are shown for a separated current and the speed for an attached current with width $w=0.1$ (circle), 0.2 (square), 0.3 (diamond), 0.4 (upright triangle), 0.5 (inverted triangle) and 0.6 (star). For the attached currents the symbol indicates the minimum $h_{b}$ for a solution.

from $R_{+}$Riemann invariant. The leading edge is joined to the rarefaction by a region of constant $h=h_{b}$ and the continuity statement $u=c_{p}$. The connection occurs at a point $x_{A}$ which has a constant speed $c_{A}\left(<c_{b}\right)$ equal to the $c_{-}$characteristic evaluated with $h_{b}$ and $u_{b}$. In the case of a gravity current the bore speed is unknown, but given a local relation $c_{b}\left(h_{b} ; \ldots\right), c_{b}$ and $h_{b}$ are obtained as part of the solution. Abbott (1961) discussed the non-rotating case with $c_{b}=\beta h_{b}^{1 / 2}$. There the Riemann invariant $R_{+}=u+2 h^{1 / 2}=2$ gives $h_{b}=4(2+\beta)^{-2}$. The bore speed $c_{b}=2 \beta(2+\beta)^{-1}$ and the junction point speed $c_{A}=(2 \beta-2)(2+\beta)^{-1}$. Similar matching of gravity current head conditions to shallow-water characteristic solutions has been explored for timedependent non-rotating flow (Garvine 1981; O’Donnell \& Garvine 1983) and for steady rotating flow (Garvine 1987).

Assuming that the flow at the gravity current head is separated from the left-hand wall as sketched in figure $1(a)$, the rotating gravity current solution is obtained by the simultaneous solution of (2.23) and the $R_{+}$invariant relation between $\bar{h}$ and $T_{e}$ from (2.17) for $\bar{h}_{b}=h_{b} / 2$ and $T_{e b}$ in the gravity current. The speed $c_{A}$ is then given from $c_{-}^{\text {sep }},(2.16)$, with $\bar{h}=\bar{h}_{b}$ and $T_{e}=T_{e b}$. The bore speed $c_{b}$ follows from either (2.24) or (2.25) used to evaluate (2.23). Between $x_{A}$ and $x_{b}$, the solutions have constant $\bar{h}=\bar{h}_{b}$ and width $w_{b}=\tanh ^{-1}\left(2 T_{e b}\right)$. For $x<x_{A}$, the flow is the rarefaction solution described above. As $w$ is decreased, eventually $w_{b}=w$. For narrower channels, the gravity current is attached to the left-hand wall $\left(w_{b}=w\right)$ and $c_{s e p}=c_{b}$. The solution procedure for $\bar{h}_{b}$ and $\hat{h}_{b}$ is the same as described above with the appropriate changes in the relations going into (2.23) and $R_{+}$from (2.18).

A schematic of the characteristic curves for the gravity current solution is given in figure $1(c)$. In the uniform gravity current region $x_{A} \leqslant x \leqslant x_{b}$, both the $c_{-}\left(=c_{A}\right)$ and $c_{+}$characteristics are uniform and $c_{+}>c_{b}$. The sketch shows the case of a separated gravity current where $0<c_{\text {sep }}<c_{A}$. If the gravity current head is attached to both walls, $c_{\text {sep }}$ is formally not defined, but it is taken to equal $c_{b}$. Provided that $\beta>1$, $c_{A}>0$ and the flow everywhere downstream of the dam is supercritical. 

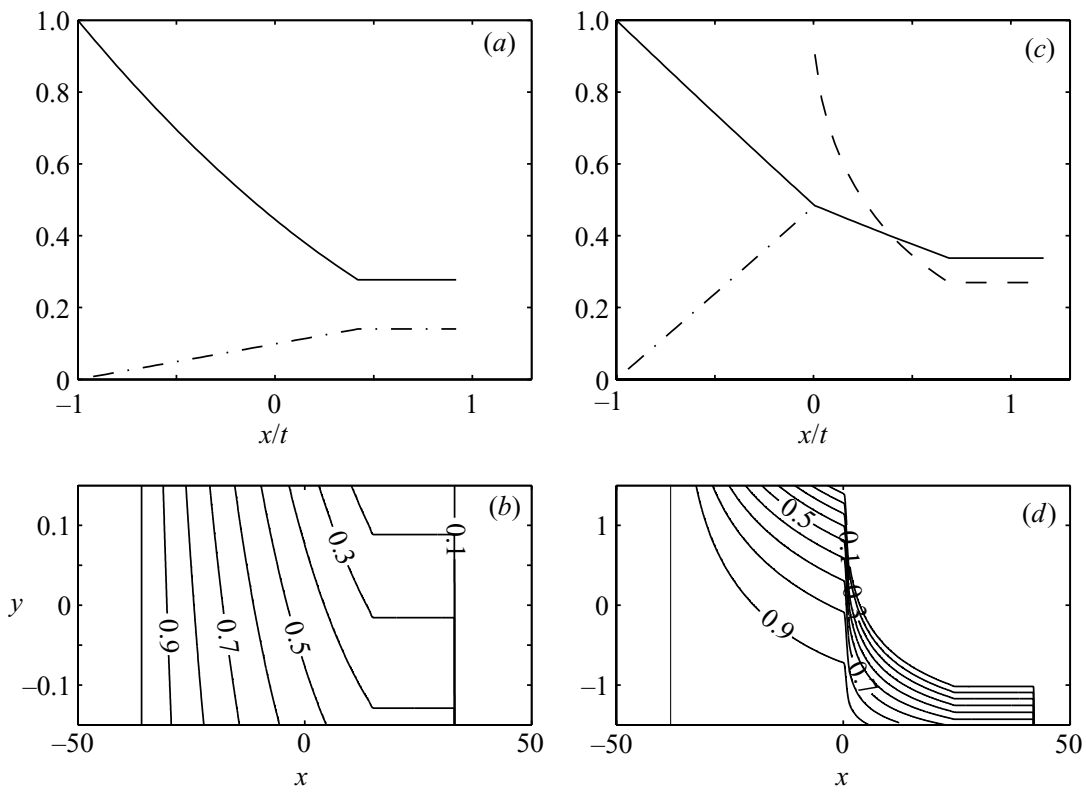

FIGURE 3. Semigeostrophic gravity current solutions $\bar{h}$ (solid line), $\hat{h}$ (dash-dot line) and $T_{e}$ (dashed line) versus the similarity variable $x / t$ for $(a) w=0.3$ and $(c) w=3$. Contour plots of the corresponding layer height fields $h(x, y)$ at $t=30$ are given in $(b) w=0.3$ and $(d) w=3$.

Examples of the solutions for an attached bore $(w=0.3)$ and a separated bore $(w=3)$ are shown in figure 3. Figures $3(a)$ and $3(c)$ show $\bar{h}, \hat{h}$ and $T_{e}$ as functions of the similarity variable $x / t$. Figures $3(b)$ and $3(d)$ are contour plots of the layer height $h(x, y)$ at $t=36$. Both examples were computed with $c_{b}$ from (2.24). Note that in this and subsequent figures, the along-channel length scale $L$ is set equal to $L_{R}$.

Solutions for $c_{b}, w_{b}$ and $h_{b}$ as functions of $w$ are given in figure 4 . The solutions are calculated for $c_{b}$ from both (2.24) and (2.25) with $\beta=1.2$. There are quantitative differences between the results for the two speed relations, but the qualitative character is the same. The bore speed and height are smallest for $w=0$, and increase monotonically with $w$. The gravity current width remains attached to the left-hand wall until $w \approx 0.4-0.5$. Once separated, $w_{b}$ grows only slightly with $w$. As anticipated, gravity currents computed with (2.24) are faster and narrower for a given $w$ than those from (2.25), provided $\beta<2^{1 / 2}$. Also shown in figure $4(a)$ is $c_{s e p}$. The critical width for separation depends on the particular $c_{b}$ relation, but once the gravity current is separated, $c_{s e p}$ is independent of $c_{b}$.

The transport at the dam, $x=0$, and in the geostrophic gravity current $Q_{b}$ are shown in figure 5. For large $w, Q_{b}$ is about half of the flux at the dam.

\section{Single-layer numerical model}

The analytical solution depends upon a number of assumptions that should be examined, particularly the imposition of the bulk gravity current head continuity constraint (2.23). This closure should not affect the solution upstream of $x_{A}$ since the flow is supercritical $\left(c_{-}>0\right)$ for $x>0$, but could result in some local effect within the gravity current. The validity of the semigeostrophic approximation in rotating dam-break problems has been explored by HKP and Stern \& Helfrich (2002) who 

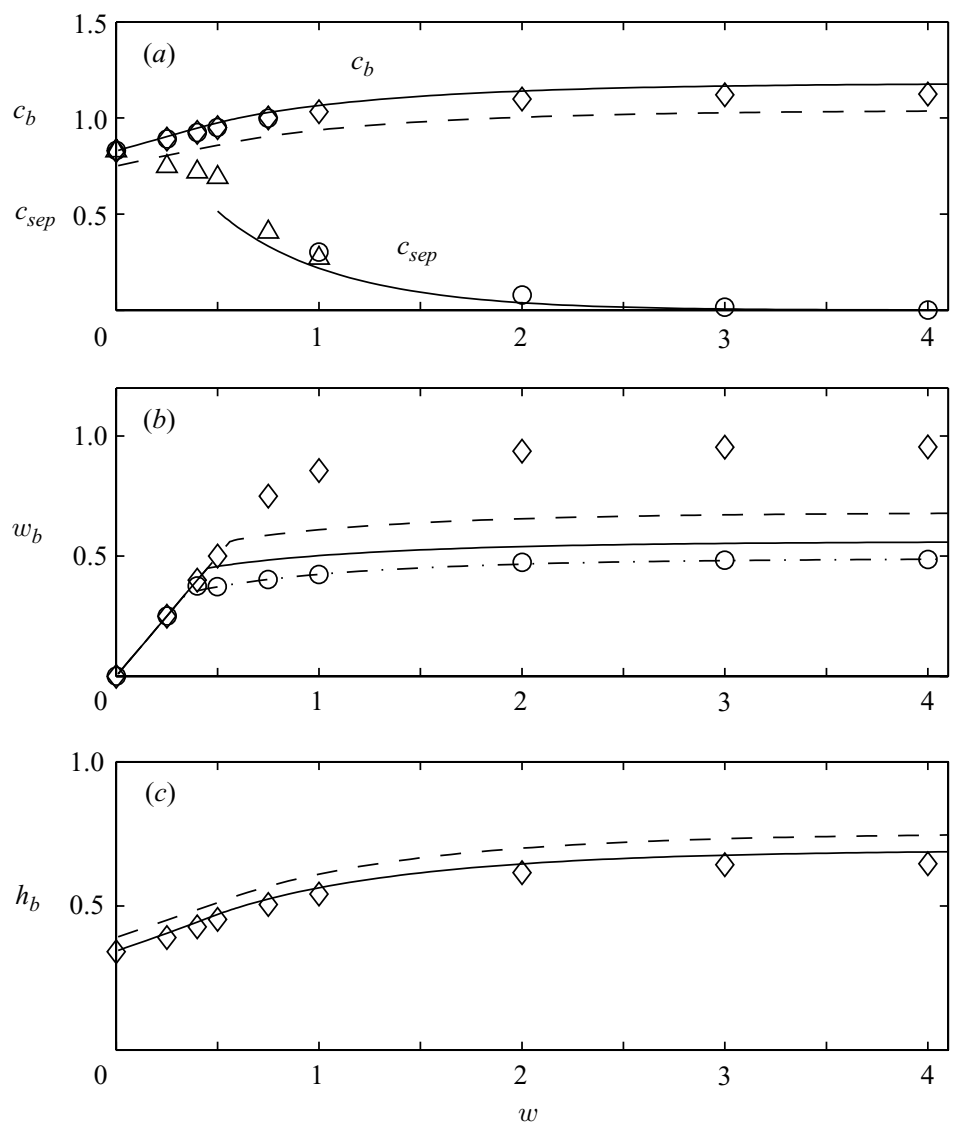

FiguRE 4. Semigeostrophic solutions for $(a) c_{b}$ and $c_{s e p}$, (b) $w_{b}$ and $(c) h_{b}$ as functions of $w$. The solid line is for $c_{b}$ from (2.24) and the dashed line from (2.25) with $\beta=1.2$. The dash-dot line in $(b)$ is the width at which the gravity current depth $h=0.1$. In $(a-c)$ the reduced-gravity numerical model results for $c_{b}, w_{b}$ and $h_{b}$ are indicated by a $\diamond$. In $(a) c_{\text {sep }}(\bigcirc)$ and $c_{\text {shock }}(\triangle)$ are shown. In $(b)$ the numerical results for the gravity widths where $h=0.1$ are indicated by a $\bigcirc$.

demonstrated generally good agreement between the rarefaction solutions theory and numerical solutions of the shallow-water equations, particularly for narrow channels or long times.

\subsection{Numerical model}

In HKP, the shallow-water equations were numerically solved in momentum flux form (i.e. with dependent variables $U=u h, V=v h$ and $h$ ). In that form, the equations do not admit a blunt bore-like gravity current because the Rankine-Hugoniot shock solution gives $c_{b} \rightarrow \infty$ as $u$ and $h \rightarrow 0$ upstream of the bore. Thus, the gravity current solutions are compared with numerical solutions of the shallow-water equations in advective form, (2.1)-(2.3) with $\delta=1$, that will naturally develop a leading bore. The solution will conserve both flow force and energy at the bore (Hacker \& Linden 2002). However, if an internal shock (connecting regions of finite depth) develops, energy, but not flow force, will be conserved across the discontinuity.

The numerical technique follows closely the finite-volume method for the conservative form of the single-layer shallow-water equations described in HKP. The method has been tested in a number of rotating flow problems involving shocks, hydraulic 


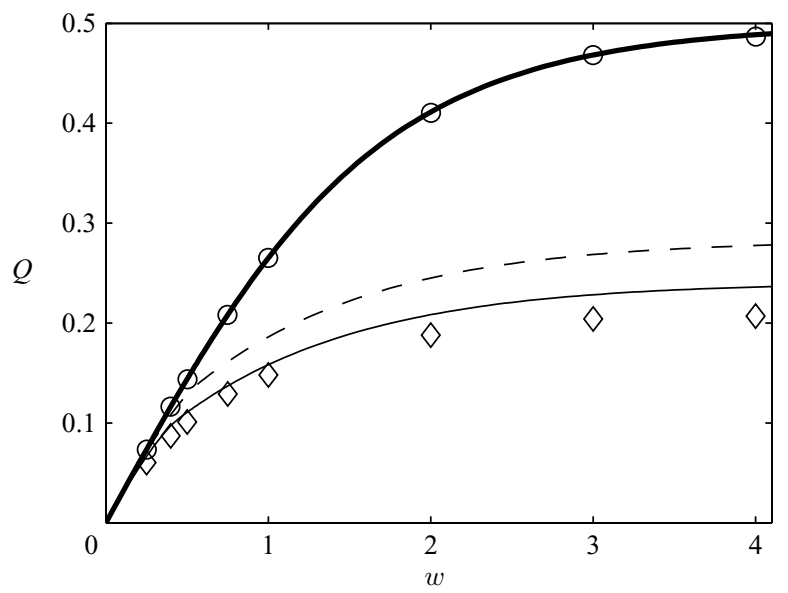

FIGURE 5. Semigeostrophic solution for the transport $Q$ at $x=0$ (thick solid line) and in the gravity current $\left(x>x_{A}\right)$ as functions of $w$. The thin solid line is for $c_{b}$ from (2.24) and the dashed line from (2.25) with $\beta=1.2$. The symbols are the reduced-gravity numerical model results for the transport in the gravity current immediately behind the gravity head $(\diamond)$ and at $x=0(\bigcirc)$.

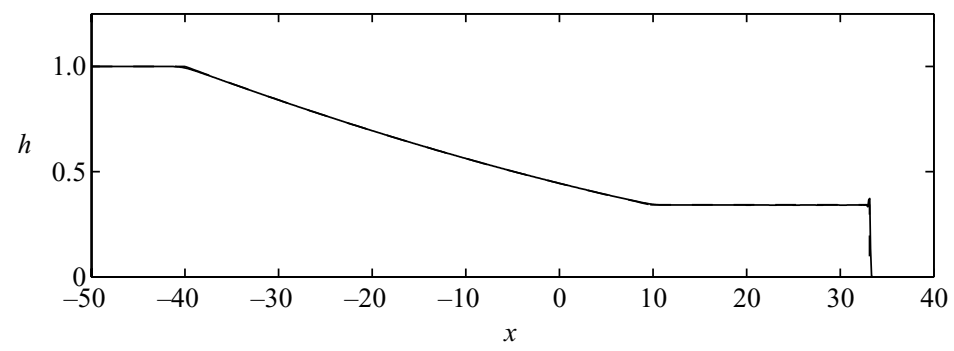

FIGURE 6. Solutions for the non-rotating $(w=0)$ case at $t=40$. Numerical model (solid) and characteristic solution with (2.24) (dashed).

jumps and the presence of zero layer depth over some part of the domain (HKP; Pratt, Helfrich \& Chassignet 2000; Stern \& Helfrich 2002). The only modification necessary for the present problem is a change in the computation of the nonlinear terms in the momentum equations from a conservative formulation to the advective form in (2.1) and (2.2) (Leveque 2002, §9.3).

The numerical model is robust, stable and does not require any explicit diffusion or friction terms. There is, of course, some numerical diffusion, but it is relatively unimportant except in certain instances discussed below. Unless specifically mentioned, no runs had explicit friction or damping terms.

The runs were all conducted in a rectangular channel of length 100 in the $x$-direction and width $w=0.125-4$. Runs were also conducted for no rotation (non-dimensional width $w=0$ ). No flux boundary conditions were employed. The calculations had grid spacing $\mathrm{d} x=0.1$ and $\mathrm{d} y=0.0125-0.05$. The layer is initialized with $\boldsymbol{u}=0$ everywhere, $h=1$ for $x<0$, and $h=10^{-8}$ for $x>0$. The layer is considered to have zero thickness if $h \leqslant 10^{-3}$, though the results are not sensitive to choices smaller than this.

\subsection{Model results}

An example of the accuracy of the numerical technique is illustrated in figure 6 for the non-rotating case, $w=0$. The agreement between the numerical and analytical 

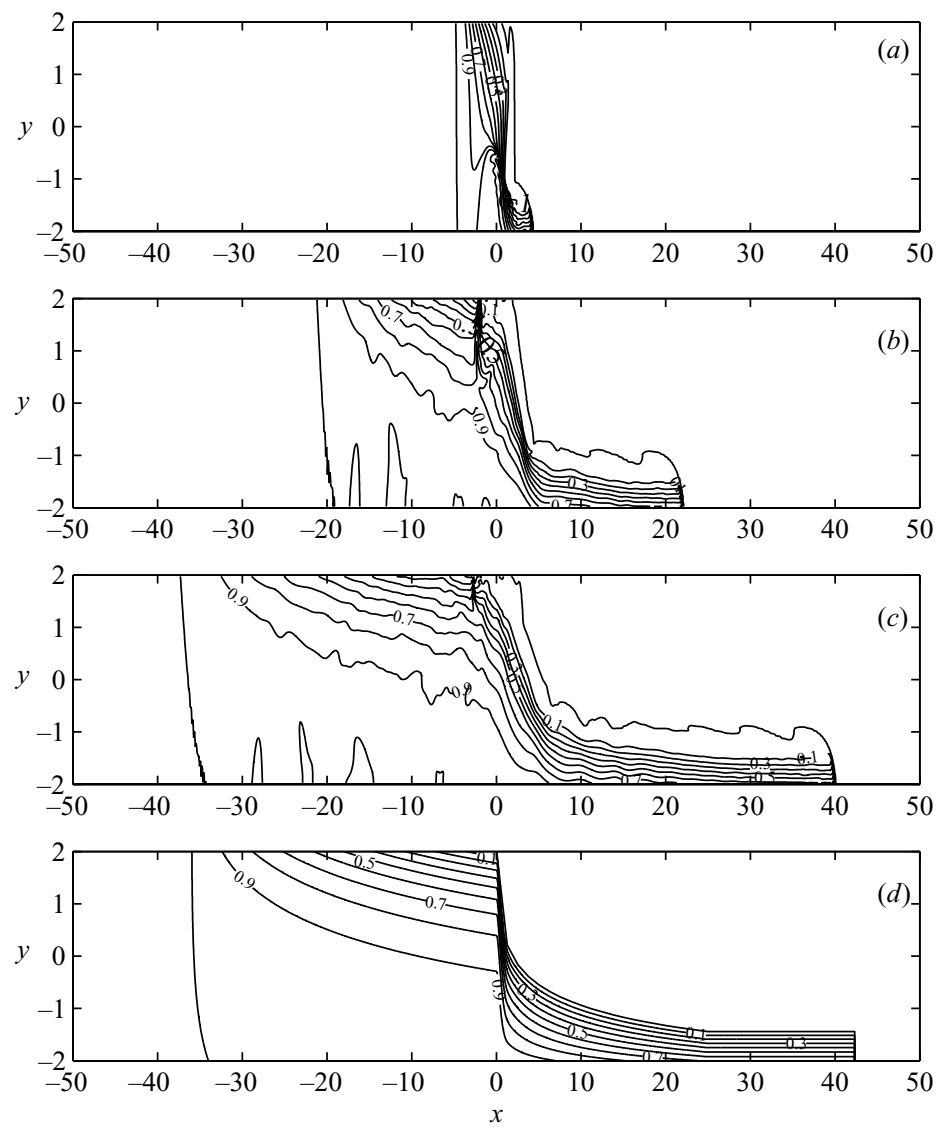

Figure 7. Numerical solution for $w=4$ at $(a) t=4$, (b) $t=20$ and $(c) t=36$. (d) The semigeostrophic characteristic solution at $t=36$. Shown are contours of the upper-layer depth $h$ at intervals of 0.1 .

solutions is very good. The bore speed $c_{b}=0.829$ and 0.828 from the numerical and analytical solutions, respectively. The corresponding $h_{b}=0.342$ and 0.343 . The greatest error is in the location of the leading edge of the upstream propagating wave and at the junction point $x_{A}$ where numerical diffusion smooths out the discontinuities in $\partial h / \partial x$. There is also some minor overshoot and oscillation at the bore.

The numerical solution for $w=4$ is shown at $t=4,20$ and 36 in figure $7(a-c)$. The semigeostrophic solution at $t=36$ is plotted in figure $7(d)$. At $t=36$ there is generally good overall agreement of the analytical and numerical solutions. The numerical model gives $c_{b}=1.124$ and $h_{b}=0.645$ at five deformation radii behind the leading edge, slightly slower and shallower than the semigeostrophic predictions $c_{b}=1.176$ and $h_{b}=0.687$. In all model runs, $c_{b}$ is constant after an initial adjustment time of $t \sim 1$. Another difference is that the numerical solution is not quite uniform in $x$ for $x>x_{A}=24.5$. In figure $7(c)$, the layer depth on the wall decreases from 0.68 to 0.65 between $x=24.5$ and 30 and to 0.64 at $x=39$. Figures $7(b)$ and $7(c)$ show that the solution near the gravity current head is nearly steady.

The greatest differences are in the region of the discontinuity of the analytical solution in the vicinity of the separation point $x_{\text {sep }} \approx 0$, and in the width of the geostrophic current trailing the gravity current head. The former is expected since 


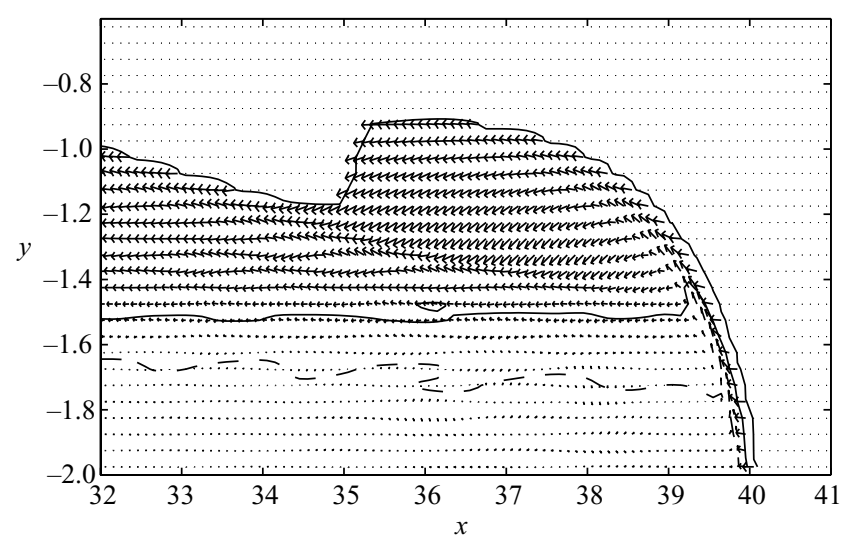

FIGURE 8. Upper-layer velocity vectors in the frame moving with the gravity current speed $c_{b}=1.0475$ from the solution in figure $7(c)$. The solids lines are the $h=0.1$ and 0.001 contours and the dashed is the $u=c_{b}$ contour.

$\delta=O(1)$ in the vicinity of $x_{\text {sep }}$. The difference in width is also linked to ageostrophic effects near the bore face ignored in the use of the gravity current head conditions to close the semigeostrophic solution. Flow in the gravity current trailing the nose is not uniform in $y$, leading to $u>c_{b}$ over some part of the gravity current. From the semigeostrophic solution (2.7), the region of flow converging on the front occurs in the offshore portion of the gravity current (the largest velocity occurs at the current edge). The flow must adjust to meet the nose condition over some region immediately behind the bore front.

In contrast, in the numerical solution, buoyant fluid within $\approx 0.3$ of the righthand wall is flowing towards the gravity current head with speed $u>c_{b}$ (figure 8). This discrepancy between theoretical and numerical velocity fields is due to potential vorticity modification within the bore face region and will be discussed below. This fluid is turned offshore to form a narrow jet of width $\approx 0.5$ immediately behind the leading edge of the bore. The jet results in a broad, thin and wavy offshore portion of the geostrophic boundary current where the fluid flows away from the head in a frame moving with the gravity current speed. This fluid could be considered as detrained from the gravity current, though it never separates from the current. The agreement in the numerical and semigeostrophic solutions of the location of the $h=0.1$ contour is much better. The flow near the head agrees qualitatively with descriptions of some of the experiments (SWH; GH; Kubokawa \& Hanawa 1984). The offshore jet is also similar in character to the ageostrophic boundary-layer jet that connects a Kelvin shock to a trailing geostrophic flow (Fedorov \& Melville 1996; HKP).

Numerical and semigeostrophic solutions for a narrow channel $w=0.4$ at $t=36$ are shown in figure 9. Again the general agreement between the two solutions is quite good. The gravity current speeds are nearly the same and the bore is attached to both walls as predicted. A significant difference, though, is the presence of a discontinuity, or shock, at $x=x_{\text {shock }} \approx 26$ in the numerical solution. The shock propagates at a constant speed $c_{\text {shock }}=0.719$. An interesting feature of the shock is that it divides the gravity current into an upstream uniform region $\left(x_{A}<x<x_{\text {shock }}\right)$ and a zone of weak gradients in $x$ behind the bore $\left(x_{\text {shock }}<x<x_{b}\right)$.

The embedded shock in figure 9 is due to the difficulty of conserving potential vorticity as fluid parcels pass through the ageostrophic boundary layer bordering the bore face. An ideal numerical model should conserve shallow-water potential vorticity 

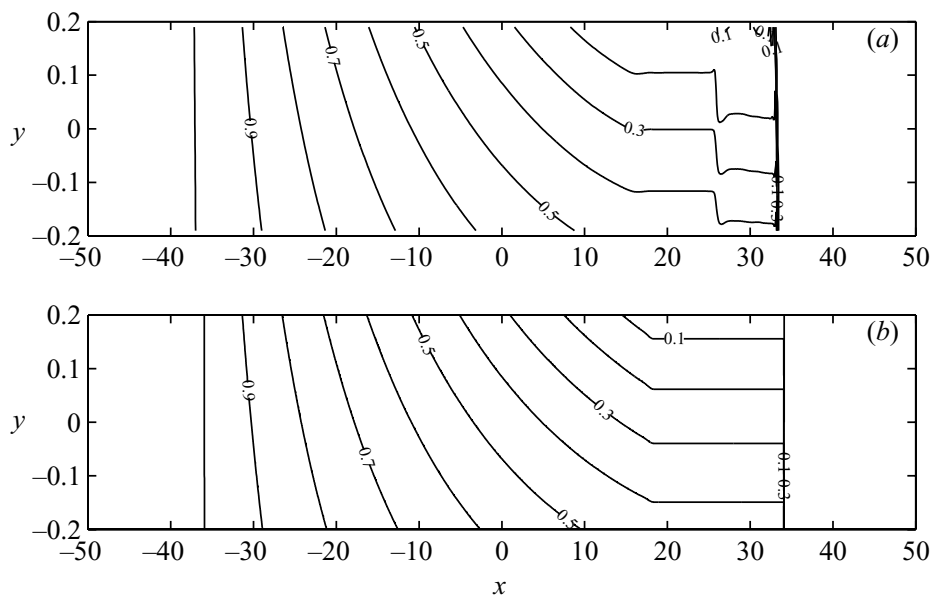

Figure 9. Contours of the upper layer depth $h$ from $(a)$ the reduced-gravity numerical solution and $(b)$ the semigeostrophic theory with $w=0.4$ at $t=36$.

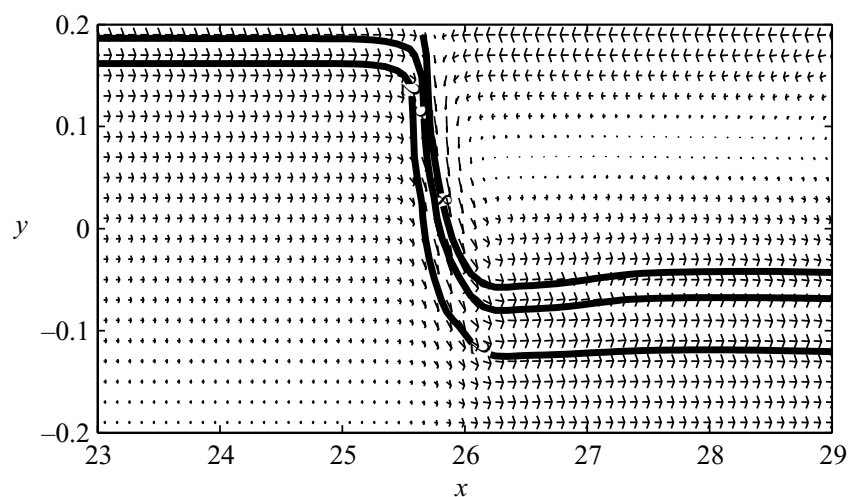

FIGURE 10. Expanded view of the velocity vectors near the embedded shock in figure 9 . The velocity vectors are in a frame moving with the shock. The thick lines are contours of potential vorticity. The values of the contours are 2,5 and 8 from the bottom to the top of the figure.

$q=\left(f+v_{x}-u_{y}\right) h^{-1}$ following fluid parcels. At the leading bore face, $h \rightarrow 0$ rapidly, consequently $f+v_{x}-u_{y}$ must approach zero at the same rate to keep $q$ constant. However, in the calculations, small errors in the lateral shear on the grid scale give large errors in $q$. Hence, as fluid parcels pass through the ageostrophic offshore jet at the bore face their potential vorticity is modified. Changes in potential vorticity can be related to dissipation (Pratt 1983; Schär \& Smith 1993), here due to numerical effects, but to be expected in general within a possibly turbulent bore.

Fluid with modified potential vorticity is pooled in the region between the shock and the bore. The potential vorticity and the velocity vectors in a frame moving with the shock in figure $9(a)$ are shown in figure 10. Fluid approaches the shock from upstream along the left-hand wall, crosses the channel within the shock, and the proceeds towards the bore along the right-hand wall. Fluid exits the bore (not shown) along the left-hand wall and flows back towards the shock, but does not pass through it. The average potential vorticity of the fluid between the bore and the shock is $q \approx 16$, much greater than the initial value of $q=1$. There is some modification of $q$ near the left-hand wall upstream of the shock (owing to the numerical boundary treatment), 

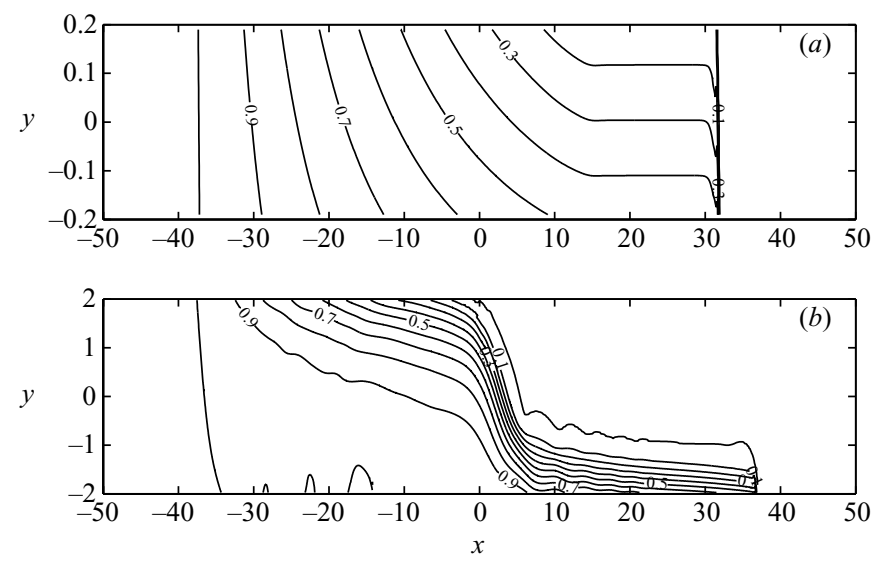

FIGURE 11. Reduced-gravity numerical solutions with lateral viscosity $v=0.005$ at $t=36$. (a) $w=0.4,(b) w=4$.

but this has a minor effect on the potential vorticity budget. The modification of the potential vorticity is due primarily to fluid parcels passing near the leading edge of the bore.

Since the potential vorticity pooled between the shock and the bore is different from the value upstream, the wave speeds are changed. For attached flow with uniform $q \neq 1,(2.13)$ is

$$
c_{ \pm}^{\text {attach }}=q^{1 / 2} \hat{h} T^{-1} \pm \bar{h}^{1 / 2}\left[1-(1-q \bar{h}) T^{2}\right]^{1 / 2},
$$

with $T=\tanh \left(q^{1 / 2} w / 2\right)$. Upstream of the shock $(q, \bar{h}, \hat{h})=(1,0.285,0.184)$, giving $c_{-}=0.41$ and $c_{+}=1.46$. Ahead of the shock, the average value $q \approx 16$ with $(\bar{h}, \hat{h})=$ $(0.261,0.168)$ gives $c_{-}=0.21$ and $c_{+}=1.82$. The convergence of $c_{-}$characteristics is consistent with a shock. Since the potential vorticity within the downstream pool is highly non-uniform, the calculation for $c_{-}$is only an estimate. A calculation of the long wave speeds on either side of the shock that accounts for the variation of the potential vorticity would presumably give a shock speed within the range of $c_{-}$. However, the speed estimates support the idea that the shock is a consequence of the modification of $q$ for fluid parcels passing along the bore face.

The shock does not occur for the non-rotating case (figure 6) since the flow is irrotational and $q$ is irrelevant. It is not clearly evident for gravity currents that are well separated from the left-hand wall (figure 7). The shock speed $c_{\text {shock }}$ approaches $c_{b}$ as $w$ decreases until the shock merges with the leading bore at $w=0$. As $w$ is increased, $c_{\text {shock }}$ approaches $c_{\text {sep }}$ from above. At $w=1$, the speed $c_{\text {shock }} \approx c_{\text {sep }}$. Beyond this width, the shock is not an identifiable feature within the strong cross-channel jet that develops near $x_{\text {sep }}$. However, the process of potential vorticity modification in the bore face region occurs for all $w$ and is the reason for the differences between the theoretical and numerical gravity current velocity structure discussed above in connection with figure 8 . Again, while the precise value of the modified potential vorticity is dependent upon the numerical model and resolution, it does represent a process (though probably not accurately) that probably occurs within a real turbulent and dissipative gravity current head.

Since the presence of the shock is linked to non-conservation of $q$, it is not surprising that it is sensitive to dissipation. Indeed, the presence of internal friction (either interfacial or lateral) eliminates the shock for sufficient dissipation. Figure 11(a) 
shows the $w=0.4$ case with Laplacian lateral friction $v \nabla^{2} \boldsymbol{u}, v=0.005$, and stressfree boundaries. At this value of $v$, the shock has merged with the bore leaving a uniform gravity current downstream of $x_{A}$. The bore speed $c_{b}$ is slightly slower and $h_{b}$ is slightly greater than the inviscid run. For the wider channel $w=4$, the gravity current width ( $h=0.1$ contour) and height now taper smoothly toward the bore (figure $11 b$ ). Again the bore travels slightly slower than the inviscid run, while $h_{b}$ just upstream of the bore is slightly smaller. In both frictional runs $c_{b} / h_{b}^{1 / 2}<2^{-1 / 2}$. As will be discussed below, laboratory experiments also typically show gradual thinning of separated gravity currents toward the nose.

The inviscid model results are summarized and compared to the semigeostrophic theory in figure 4 . The speeds $c_{b}$ and $c_{s e p}$ from the model agree well with the predictions of the semigeostrophic solution with $c_{b}$ from (2.24) (figure $4 a$ ). Also shown is the speed of the shock $c_{\text {shock }}$ when it is clearly identifiable. The most significant exception for $c_{\text {sep }}$ is near the transition from attached to separated flow. The broader gravity current produced by the offshore jet at the gravity current head causes separation to occur for a wider channel $(w>0.075)$ than the theoretical prediction $w=0.46$. For separated gravity currents, the widths $w_{b}$ are substantially greater than the predicted (figure $4 b$ ). The width of the gravity current from the wall to the $h=0.1$ contour agrees well with the theoretical prediction. Despite the significant variations in potential vorticity, the bore speeds $c_{b}$ and heights $h_{b}$ fall only slightly below the theory with increasing $w$, but $\beta=c_{b} / h_{b}^{1 / 2} \approx 1.41$ is nearly constant. This is perhaps not surprising as $\mathrm{SWH}$ showed that the bore properties were relatively insensitive to changes in $q$.

The smaller $h_{b}$ in the numerical solutions are also reflected in the gravity current transports shown in figure 5. This is expected since from geostrophy $Q_{b}=h_{b}^{2} / 2$ for separated flows. The numerically computed transports at the dam $(x=0)$ are in good agreement with the theory, as found previously in HKP.

\section{Laboratory experiments}

Of the existing laboratory experiments only those of SWH are easily compared with the theory. GH used both a uniform-width channel and a channel with different widths ahead and behind the dam. More crucially, it is possible only for a few situations to link their results (e.g. $w_{b}$, etc.) to the basin parameters. An exception is data from their figure 4 that will be compared to a theory for bore speed decay in $\S 6$. Kubokawa \& Hanawa (1984) had the gravity current flow out into a channel with a non-constant width and had a sill at the dam location that produced a vigorous two-layer exchange flow there that is beyond the applicability of the model.

The SWH experimental results to be considered are the 14 runs in their table 1 . They were all conducted in a $20 \mathrm{~cm}$ wide channel. The dammed region occupied $49 \mathrm{~cm}$ at one end of the $183 \mathrm{~cm}$ long channel. The total fluid depth varied from run-to-run, but was in the range $H_{T}=20-22 \mathrm{~cm}$. Their $g^{\prime}=2.1-17.15 \mathrm{~cm} \mathrm{~s}^{-2}, H=4-8.7 \mathrm{~cm}$, and $f=0.21-0.87 \mathrm{~s}^{-1}$. The non-dimensional channel width $w=0.94-3.7$ and

$\sqrt{g^{\prime} H}=2.5-8.8 \mathrm{~cm} \mathrm{~s}^{-1}$. These experiments did not examine small $w$ where the theory predicts the gravity current remains attached to both walls. Nor did they report observations of the separation point speed $c_{\text {sep }}$. Thus an additional suite of experiments was undertaken to fill these gaps.

\subsection{Experimental method}

The experiments were carried out on a new $1 \mathrm{~m}$ diameter rotating table in the geophysical fluid dynamics laboratory at the Woods Hole Oceanographic Institution. 


\begin{tabular}{ccccccccccc}
\hline Run & $w$ & $\begin{array}{c}H \\
(\mathrm{~cm})\end{array}$ & $\begin{array}{c}H_{T} \\
(\mathrm{~cm})\end{array}$ & $\begin{array}{c}g^{\prime} \\
\left(\mathrm{cm} \mathrm{s}^{-2}\right)\end{array}$ & $\begin{array}{c}f \\
\left(\mathrm{~s}^{-1}\right)\end{array}$ & $\begin{array}{c}L_{R} \\
(\mathrm{~cm})\end{array}$ & $\begin{array}{c}c_{b}^{*} \\
\left(\mathrm{~cm} \mathrm{~s}^{-1}\right)\end{array}$ & $\begin{array}{c}h_{b}^{*} \\
(\mathrm{~cm})\end{array}$ & $\begin{array}{c}w_{b}^{*} \\
(\mathrm{~cm})\end{array}$ & $\begin{array}{c}c_{s e p}^{*} \\
\left(\mathrm{~cm} \mathrm{~s}^{-1}\right)\end{array}$ \\
1 & 4.02 & 4.4 & 28.9 & 4.95 & 1.25 & 3.73 & 4.3 & 2.7 & 1.8 & 0.2 \\
2 & 3.11 & 4.5 & 28.5 & 5.16 & 1.00 & 4.81 & 3.8 & - & 2.6 & 0.2 \\
3 & 1.95 & 4.7 & 28.6 & 12.56 & 1.00 & 7.68 & 6.3 & 2.2 & 3.4 & 0.5 \\
4 & 0.99 & 4.6 & 28.5 & 12.52 & 0.50 & 15.18 & 6.2 & 2.7 & 5.7 & 2.0 \\
5 & 0.50 & 5.9 & 28.8 & 18.35 & 0.35 & 29.72 & 8.7 & 2.8 & 10.3 & 4.1 \\
6 & 0.25 & 6.1 & 28.8 & 13.53 & 0.15 & 60.55 & 8.6 & 2.3 & 15.0 & 3.8
\end{tabular}

TABLE 1. Summary of experimental runs. The width of the annulus was $15 \mathrm{~cm}$ in all runs. The asterisk superscript denotes the dimensional form of a variable.

The table is driven by a direct drive d.c. torque motor. Digital control of the table position results in speed variations of less than $0.02 \%$ of the set rotation rate. A cylindrical acrylic tank with internal diameter of $0.965 \mathrm{~m}$ and depth $0.418 \mathrm{~m}$ was fitted with a concentric inner polycarbonate sheet barrier to form an annulus of width $0.15 \mathrm{~m}$. The inner wall was fixed in place $1 \mathrm{~cm}$ above the tank bottom to ensure that pressures on either side of the barrier were equal. The water in the inner cylinder played no active role in the experiments and its only purpose was to hold the barrier in shape. A quarter of the annulus was isolated between a fixed vertical endwall and a removable vertical plate to form the upstream basin.

The tank was filled with saltwater of density $\rho_{2} \approx 1.020$ to a depth slightly less than the total depth $H_{T}$. The tank was then spun-up to a counterclockwise rotation rate $f / 2$. The dam was inserted and buoyant water $\left(\rho_{1}\right)$, dyed blue for visualization, was then added behind the dam with a floating foam diffuser to form a layer of depth $H$. Additional time was allowed to achieve near solid-body rotation and $H_{T}$ was then recorded. Densities were measured using an Anton Paar model DMA58 densitometer with accuracy of $10^{-5} \mathrm{~g} \mathrm{~cm}^{-3}$.

A summary of the experimental parameters for the six runs spanning $w=0.25-4.02$ is given in table 1 . In all cases, $H / H_{T} \leqslant 0.21$ so that the lower-layer motion should be weak and the reduced-gravity approximation reasonable.

The experiments were visualized with two co-rotating video cameras. One was positioned above the tank for a plan view of the flow. From these images, quantitative observations were made of the bore position $x_{b}$ and separation point $x_{\text {sep }}$, and the bore width $w_{b}$. The second camera provided a side-view image of the gravity current through the outside tank wall (right-hand wall) about $95 \mathrm{~cm}$ downstream of the dam from which $h_{b}$ was found. The video images were digitized directly to a computer at specified intervals ( $1 / 6$ to $2 \mathrm{~s}$ ). Additional side-view images were taken with a digital $35 \mathrm{~mm}$ camera fixed in the lab frame.

\subsection{Experimental results}

In this and the following sections, some results will be presented using dimensional variables which will be distinguished by a superscript asterisk from their nondimensional versions.

Plan-view images of the gravity currents for $w=0.25,0.99$ and 4.02 are shown in figure 12, along with a side-view image of the gravity current head for $w=0.99$. All the images were taken about half-way through a run. The images show that the separation point $x_{\text {sep }}^{*}$ remains closer to the dam with increasing $w$, as expected. Aside from the indications of turbulent mixing, the most obvious difference between the images and the theory and numerical results of the previous sections is that the 
(a)

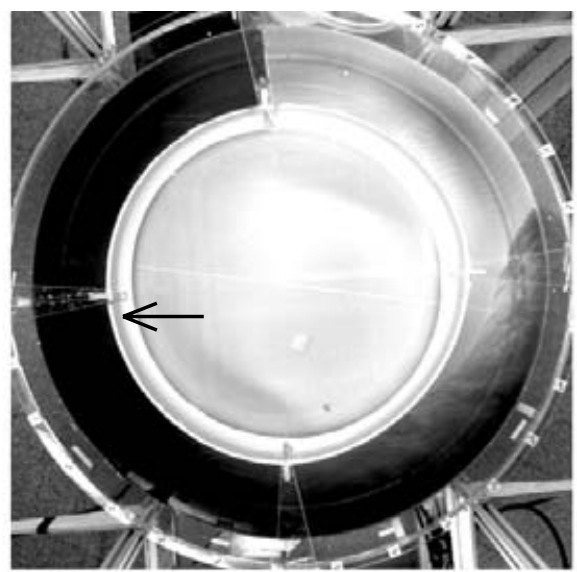

(b)

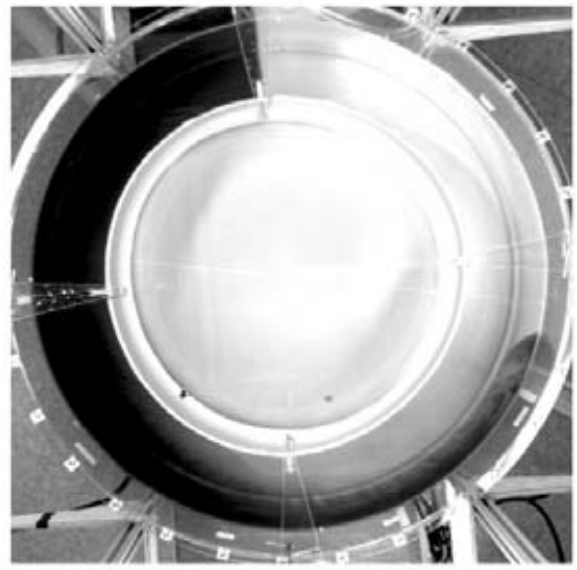

(c)

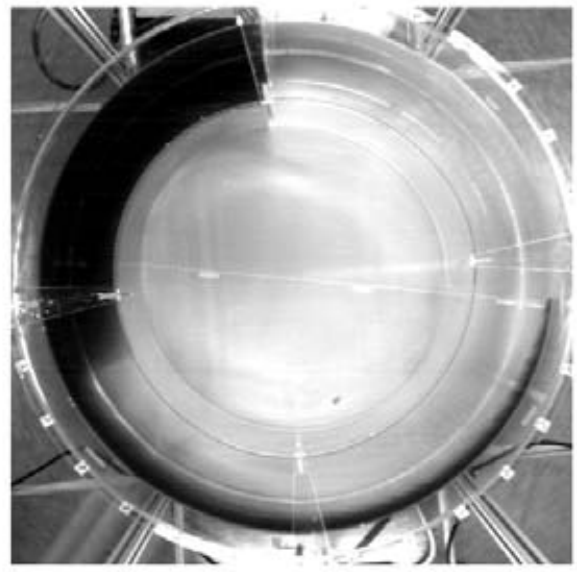

(d)

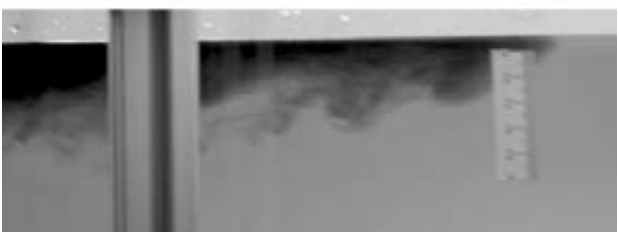

FIGURE 12. Plan view images of the experimental runs for $(a) w=0.25, t^{*}=18 \mathrm{~s} ;(b) w=0.99$, $t^{*}=20 \mathrm{~s}$; and $(c) w=4.02, t^{*}=45 \mathrm{~s}$. The dam location is indicated by the arrow in $(a)$. (d) Sideview image of gravity current head for $w=0.99$ taken several seconds before the image in $(b)$. 


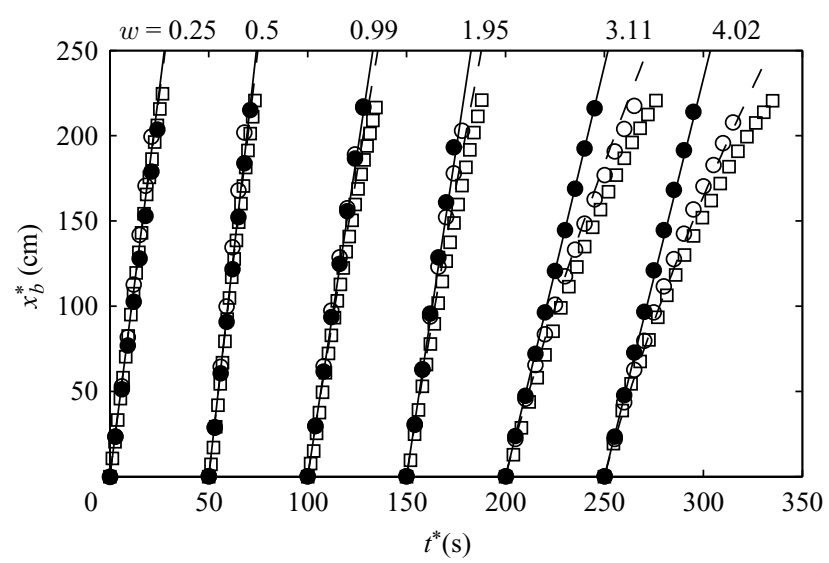

FIGURE 13. Dimensional gravity current head position $x_{b}^{*}$ versus $t^{*}$. Experimental data ( $\square$ ), annular domain ROMS model with slip walls $(\bullet)$ and no-slip walls $(O)$. The solid line is the trajectory with speed $\sqrt{g^{\prime} H}$ for each case. The dashed line is from the viscous drag theory (6.4) with $U_{0}=1$ and $a=2.8$. The non-dimensional channel width $w$ is indicated. Starting times are offset by $50 \mathrm{~s}$.

gravity current width (here defined as the total width of dyed fluid) continually thins as the head is approached. It is particularly evident in figure 12(a) where the theory and two-layer numerical model have the head attaching to each wall at the same location, i.e. $x_{\text {sep }}^{*}=x_{b}^{*}$. This is due in part to the annular channel which in this case has an inner radius $<L_{R}$. However, the difference between $x_{\text {sep }}^{*}$ and $x_{b}^{*}$ in figure 12(a) is less than $L_{R}$ and thus is not resolved by bulk closure (2.23) used in the theory.

The gravity current head position $x_{b}^{*}$ is plotted against $t^{*}$ in figure 13. As in the previous experiments, some of the trajectories are noticeably curved, indicating decreasing $c_{b}^{*}$ with time. Here it is clear that this effect becomes more pronounced as $w$ increases. These data can be fitted reasonably well with the exponential model

$$
x_{b}^{*}=X_{M}^{*}\left(1-\mathrm{e}^{-t^{*} / \tau^{*}}\right)
$$

employed by GH. Here, $\tau^{*}$ is a decay time scale and $X_{M}^{*}$ is the length scale at which the gravity current would stagnate. However, there were no indications of stagnation of the plume head and gyre formation as reported by SWH. The corresponding Reynolds numbers and other experimental parameters are comparable to those in SWH.

GH found that the non-dimensional decay time $\tau=\tau^{*} f$ data could be collapsed, after multiplication with $F r^{-2 / 3}=\left(g^{\prime} H / f^{2} A_{0}\right)^{1 / 3}$, when plotted against the Ekman number $E=v / f H^{2}$ (their figure 10). Here, $A_{0}$ is the horizontal area of the dammed region and $\mathrm{Fr}$ was taken to be a Froude number related to the velocity of fluid leaving the reservoir. For $E<10^{-3}, \tau \mathrm{Fr}^{-2 / 3}$ was essentially constant and for larger $E$ it fell off as $E^{-1}$. However, unless the basin is so small that the upstream-propagating Kelvin wave could travel around the upstream basin and catch up with the gravity current, $A_{0}$ should not enter the problem. For the new experiments, the time for the Kelvin wave to circuit the upstream basin and return to the dam is longer than the first indications of nonlinear behaviour in $x_{b}$. Furthermore, the semigeostrophic solution velocity field at $x=0$ is constant in time and depends only on $w$. GH argued that the speed decay for runs with $E<10^{-3}$ is due to drag from Taylor column formation and inertial wave radiation into the lower layer. For $E>10^{-3}$, they attributed the decay 


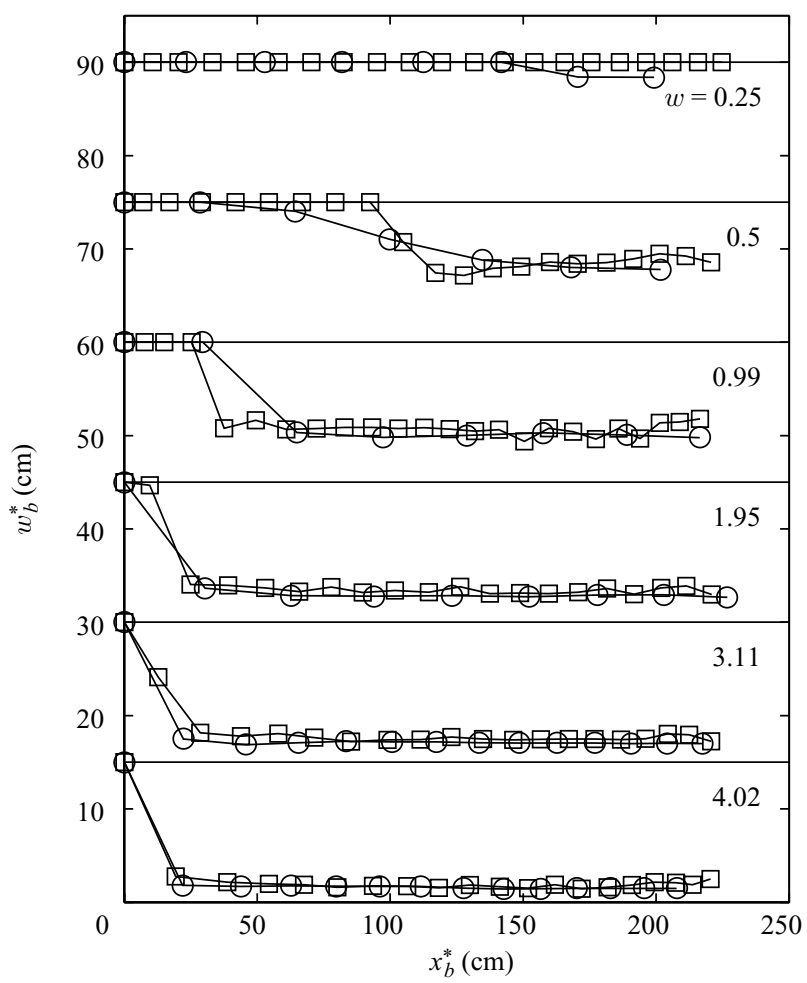

FiguRE 14. Dimensional gravity current width $w_{b}^{*}$ vs. $x_{b}^{*}$ from the experiments (squares) and annular domain ROMS model runs with no-slip walls (circles). The width is measured at $x_{\text {nose }}^{*}-1.5 L_{R}$. The non-dimensional channel width $w$ is indicated.

to Ekman processes. They did not, however, provide a prediction for $\tau^{*}$ apart from their scaling of the data. Furthermore, in the Ekman regime, $\tau$ should scale as $E^{-1 / 2}$, not $E^{-1} \mathrm{Fr}^{2 / 3}$. The present experiments give $\tau \sim R e^{2 / 3}$, where $R e=\left(g^{\prime} H\right)^{1 / 2} L_{R} / \nu$. With only six points this correlation is not conclusive, but does suggest that lateral friction is important. The speed decay will be explored further in $\S \S 5$ and 6 .

There is no clear choice for the gravity current width from the experimental results owing to the tapering plume width as the head is approached. To make a systematic comparison possible, the width $w_{b}^{*}$ was taken as the width of the dyed fluid $1.5 L_{R}$ behind $x_{b}^{*}$. This definition is similar to GH and Kubokawa \& Hanawa (1984), but differs from SWH who defined $w_{b}$ to include only fluid with $u>c_{b}$. The observed $w_{b}^{*}$ is shown versus $x_{\text {nose }}^{*}$ in figure 14 . The width reaches a nearly constant value after a distance $2-3 L_{R}$ from the dam. Different choices for the point at which the width plume is measured give similar results (but with different values for $w_{b}$ ) indicating that in the neighbourhood of the gravity current nose, the surface shape of the plume is nearly constant. When images from different times are shifted to align the plume nose, the shapes are nearly identical. The region of similarity grows as time increases.

The new experimental data and those from SWH are compared with the semigeostrophic theory in figure 15. The theoretical curves are computed with $c_{b}$ from (2.25) and the nominal value $\beta=1.2$. In view of the nonlinear behaviour of $x_{b}$, the experimental values of $c_{b}$ in figure 15(a) (and given in table 1) are computed by fitting a line to the $x_{b}$ data from $5 H / L_{R}<t<12$. The lower limit is the initial 

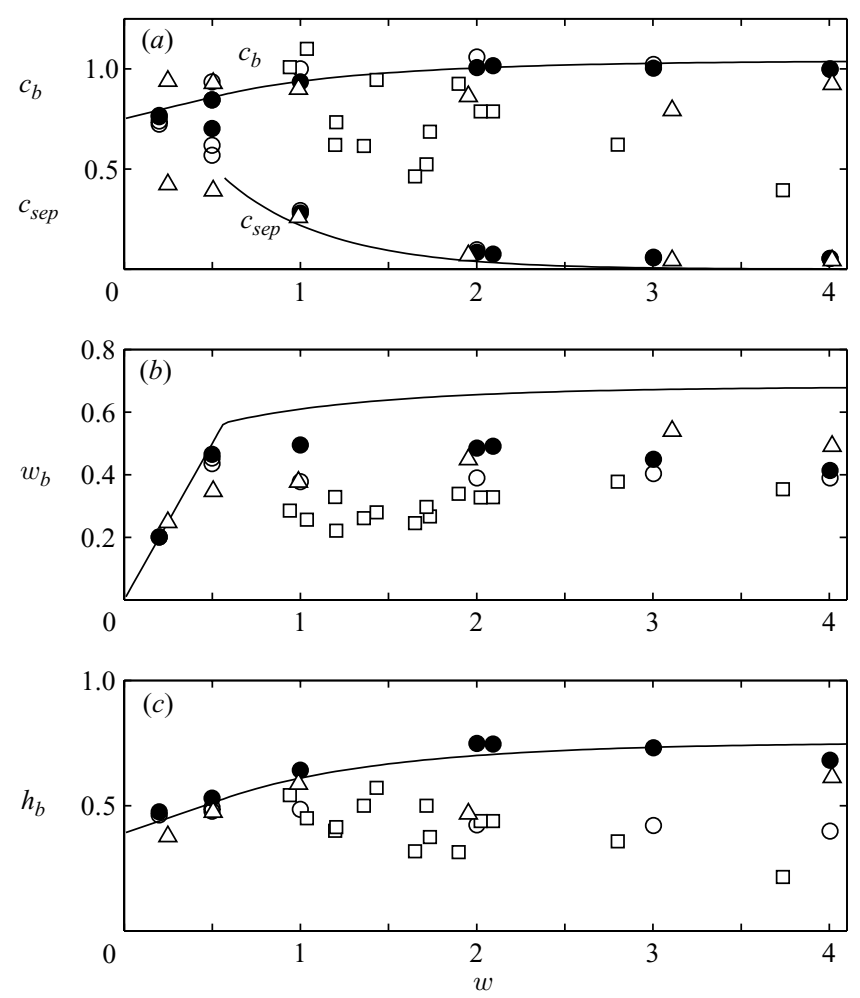

FIGURE 15. Comparison of the semigeostrophic solutions for $(a) c_{b}$ and $c_{\text {sep }}$, (b) $w_{b}$ and $(c)$ $h_{b}$ with laboratory experiments and the ROMS numerical model. The solid lines are for $c_{b}$ from the laboratory result (2.25) with $\beta=1.2$ The triangles are data from the new laboratory experiments and the squares are from the SWH experiments. The ROMS model results for the straight channel are shown for slip walls (filled circles) and no-slip walls (open circles).

gravitational adjustment time scale found by GH. The upper limit gives an estimate of $c_{b}$ before viscosity significantly affects the evolution. SWH also calculated $c_{b}$ from head displacement at early times. These new data generally fall above the SWH values without the pronounced tendency to decrease with increasing $w$. They do fall slightly above the theoretical curve for small $w$ and below for larger $w$, but overall, the agreement is satisfactory considering the assumptions in the theory and the experimental uncertainly.

The experimental values for $c_{\text {sep }}$ are also shown in figure 15(a). The $x_{\text {sep }}(t)$ data from which they are computed are somewhat noisier than the $x_{b}$ data, but do not display the nonlinear trend. The experiments agree well with the theory for $w \geqslant 1$. For lower values of $w$, where the theory predicts $c_{b}=c_{\text {sep }}$, the experiments have a cross-channel tilt of the gravity current head (cf. figure 12) which increases in time. While some of this can be attributed to the annular geometry, $c_{s e p}<c_{b}$ for small $w$ also occurs in numerical solutions in rectangular channels $(\S 5)$.

Gravity current width $w_{b}$ is shown in figure $15(b)$. The plotted points (given in table 1) are the average widths in figure 14 after the initial adjustment period. Both the new data and the SWH results fall below the theoretical prediction, though both display the weak tendency for $w_{b}$ to increase with $w$ for $w>0.6$. SWH's definition of $w_{b}$ explains the lower values in comparison to the new experiments. The non-uniform 
width of the gravity currents is also a factor in the lower widths compared to the theory. Measurements of $w_{b}$ further behind the bore nose improve the agreement.

$\mathrm{GH}$ distinguish between the maximum wall depth of the bore head and the depth of the trailing geostrophic current. They found that the former was about $30 \%$ larger. Both decayed with time, but $c_{b}^{*} /\left(g^{\prime} h_{b}^{*}\right)^{1 / 2}$ remained a constant. The present data for $h_{b}$ shown in figure 15(c) were obtained at a fixed distance downstream of the dam and immediately behind the head. SWH found $h_{b}$ by taking the average in $x$ and $t$ of the wall depth behind the bore head. The new experiments are in reasonable agreement with the theory for $w \leqslant 1$ and fall below the theory for larger $w$, as do the SWH data. The experiments with increasing $w$ tend to last longer in non-dimensional time $t^{*} f$, allowing more opportunity for dissipative effects to become important.

\section{Primitive equation numerical model}

While the theory agrees qualitatively with the experiments, there are significant quantitative differences that require explanation. The most likely sources for the differences arise from the frictional and turbulent nature of the experiments which are beyond the theory. The single-layer calculations have already indicated how nonconservation of potential vorticity (i.e. dissipation) can significantly alter the flow. An additional consideration is the continuous stratification of the experiments. The experimental bore heights were just several $\mathrm{cm}$, comparable to the initial $\sim 1 \mathrm{~cm}$ thickness of the stratified interface between the layers. The finite lower layer may also play a role, though the ratio $h_{b} / H_{T}<0.1$ and lower-layer effect should be minimal (Huppert \& Simpson 1980). The disagreement between the experiments and theory is further explored through numerical solutions of the three-dimensional hydrostatic primitive equations which include these effects. One deficiency of such a model is that mixing resulting from small-scale non-hydrostatic effects is not explicitly captured. However, the role of turbulent mixing can be assessed through the differences between the model and experiments.

\subsection{Model and set-up}

The model used is the Regional Ocean Modeling System (ROMS) model (Shchepetkin $\&$ McWilliams 2003). The principal model features used in the present calculation are the third-order upwind advection scheme for both density and momentum which is advantageous in resolving the large gradients at the gravity current head. The model has a free surface and can incorporate a number of different vertical mixing schemes, though all the calculations presented here used Laplacian diffusion in the vertical and horizontal with isotropic and constant coefficients.

The model was run in two configurations. The first was a rectangular channel $300 \mathrm{~cm}$ long and either 10 or $20 \mathrm{~cm}$ wide. This domain is similar to the $\mathrm{SWH}$ experimental conditions which facilitates comparison with the theory. The domain was filled with $20 \mathrm{~cm}$ of still water with density $\rho_{2}$ in the region ahead of the dam and with $4 \mathrm{~cm}$ of water with density $\rho_{1}$ above $16 \mathrm{~cm}$ of $\rho_{2}$ water behind. The dam was located $70 \mathrm{~cm}$ from one end of the channel. The initial density interface was a $\tanh \left(\lambda z^{*}\right)$ shape with $\lambda=0.25 \mathrm{~cm}^{-1}$ to produce an interface with thickness $\sim 1 \mathrm{~cm}$. The diffusion coefficients are set to the molecular values for momentum and salt of $10^{-2}$ and $1.3 \times 10^{-5} \mathrm{~cm}^{2} \mathrm{~s}^{-1}$, respectively. However, because of numerical diffusion, the actual coefficients, particularly the salt coefficient, are likely to be somewhat larger. The horizontal domain uses a rectangular grid with $\mathrm{d} x=1 \mathrm{~cm}$ and $\mathrm{d} y=0.25 \mathrm{~cm}$. The vertical grid is stretched to concentrate about half of the 30 grid points in the upper $5-8 \mathrm{~cm}$. 
The numerical experiments had $g^{\prime}=6.24 \mathrm{~cm} \mathrm{~s}^{-1}$. With $H=4 \mathrm{~cm}, L_{R}$ was varied by changing $f$ to give runs $w=(0.2,0.5,1,2,3,4)$. Several runs with different $g^{\prime}$, but $w$ in the range above, were also carried out. The model was run with both slip and no-slip conditions at the vertical basin boundaries.

The second configuration was an annulus with the dimensions of the laboratory apparatus in $\S 4$. The orthogonal grid had 303 equally spaced points in the azimuthal direction, 60 in the radial, and 30 in the vertical with the same upper-layer concentration. The runs were set up with the experimental parameters given in table 1 . Runs were made for slip and no-slip lateral walls.

\subsection{Model results}

Three annular channel runs with $w=0.25,0.99$ and 4.02 and no-slip conditions are shown in figure 16 at the same times as the experimental images in figure 12 . The plan-view plots show contours of

$$
\tilde{h}=H^{-1} \int_{-H_{T}}^{0} \frac{\rho(r, \theta, z)-\rho_{1}}{\Delta \rho} \mathrm{d} z,
$$

which is a measure of the non-dimensional upper-layer depth. The side views are contours of $\left(\rho-\rho_{1}\right) / \Delta \rho$ on the outer wall of the channel.

The plan-view plume structure in figures $16(a-c)$ agree quite well with the experimental images in figure $12(a-c)$. The gradual tapering of the plume width towards the nose is reproduced in the model as well as the variation of $x_{s e p}^{*}$ with $w$. Neither the model nor the experiments show indications of baroclinic instability of the gravity current. The main differences are the greater gravity current propagation distances and the apparent absence of turbulent mixing in the model results. The irregularities in the density fields on the outer annulus wall are indicative of density overturns. These may be a manifestation of Kelvin-Helmholtz instability at the hydrostatic limit or they may be a consequence of the inability of the model to adequately capture the large gradients in density and velocity at the gravity current head. In either case, the result is to produce some mixing in the vicinity of the gravity current head.

Runs with slip boundary conditions are qualitatively similar, but have larger gravity current propagation distances than the no-slip runs. This is illustrated in figure 13 where $x_{b}^{*}$ from the annular channel model is plotted along with the experimental data. The slip wall boundary condition results in a nearly linear relation between $x_{b}^{*}$ and $t^{*}$, and thus a constant bore speed, for all $w$. The no-slip boundary condition, on the other hand, give nonlinear $x_{b}^{*}\left(t^{*}\right)$ trajectories similar to the experiments. The numerical results have a slightly larger decay time scale $\tau^{*}$ than the experiments. However, for the experiments with $w \geqslant 1.95$ where the speed decay is clear, the difference in $x_{b}^{*}$ between the experiments and the slip boundary condition results is reduced by about $80 \%$ with the no-slip conditions.

The values of $w_{b}^{*}$ at $1.5 L_{R}$ behind $x_{b}^{*}$ from the no-slip annular channel model runs are plotted along with the experimental data in figure 14. The numerical model tends to give slightly lower values for $w_{b}^{*}$, but otherwise the agreement is very good.

Despite the lack of resolved turbulent mixing in the numerical model, the overall agreement with the experiments is good. Thus, the model can be used with some confidence to help explain the differences between the laboratory experiments and the semigeostrophic theory in figure 15.

The results for $c_{b}, c_{\text {sep }}, w_{b}$ and $h_{b}$ from the ROMS model in the rectangular channel with both slip and no-slip lateral boundary conditions are included in figure 15 . The straight channel runs remove effects of the annular geometry, facilitating direct 
(a)
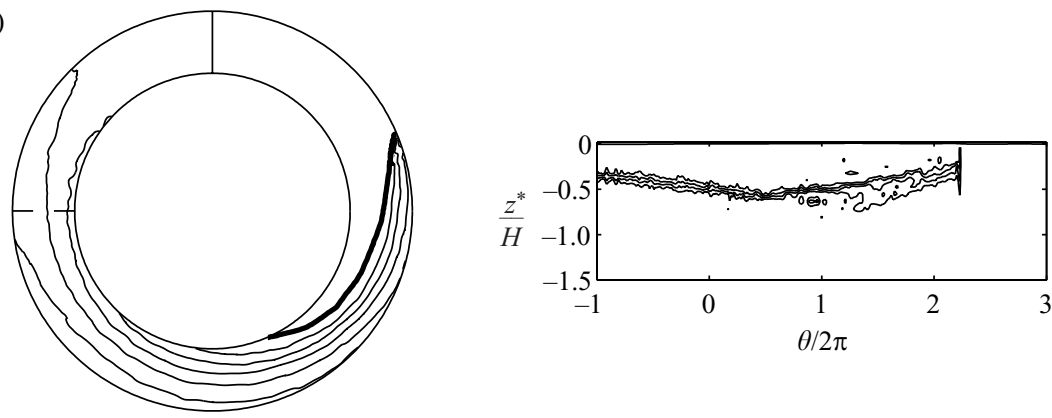

(b)
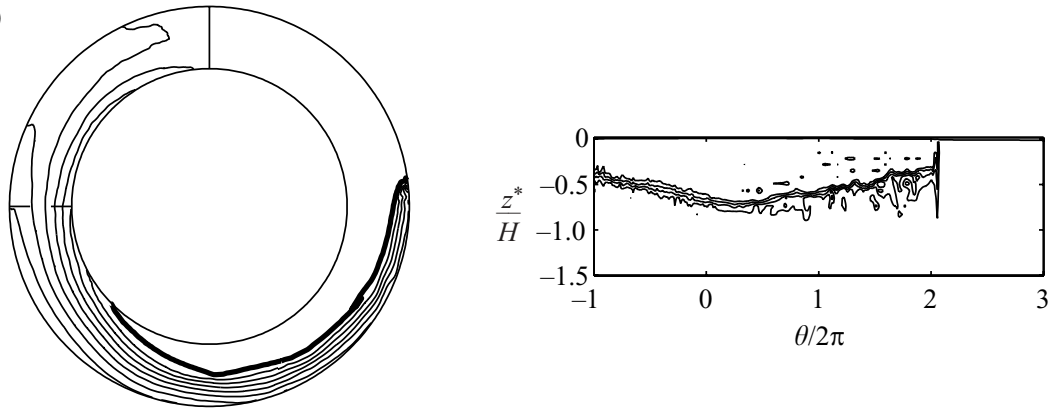

(c)
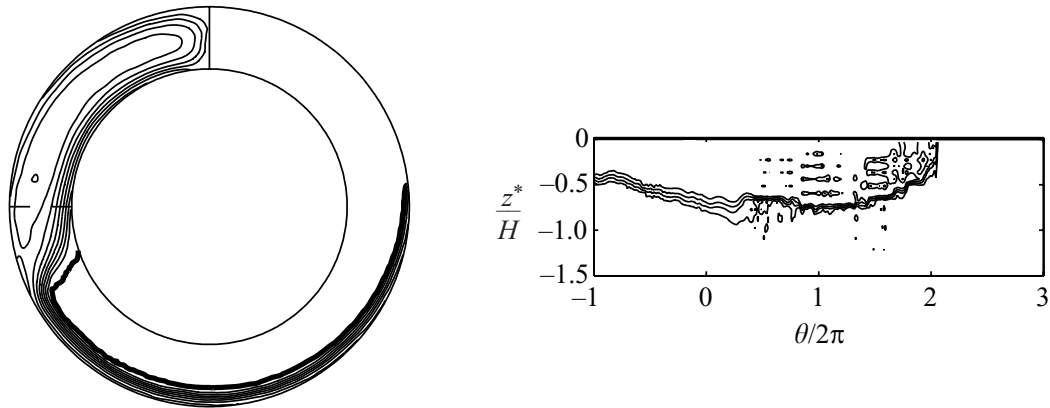

FIGURE 16. ROMS model runs with the annular domain and experimental parameters for $(a)$ $w=0.25, t^{*}=18 \mathrm{~s} ;(b) w=0.99, t^{*}=20 \mathrm{~s} ;(c) w=4.02, t^{*}=45 \mathrm{~s}$. The left-hand column shows contours of $\tilde{h}$ at intervals of 0.1 . The heavy (0.001) contour defines the plume edge. The plume density anomaly contours (interval of 0.2 ) on the outside wall are shown in the right column. In the left-hand column the dammed basin occupies the upper left quadrant, bounded by the dam (dashed line) and the back wall (solid line). The dam is at $\theta=0$ in the right-hand column.

comparison with the theory and the SWH experiments. As with the annular channel runs, $c_{b}$ is nearly constant for slip boundary conditions and decays slowly for no-slip conditions. Values for $c_{b}$ shown in figure 15(a) are the initial speeds calculated in the same manner as the experimental data. Both the slip and no-slip cases agree well with the theory computed from (2.25) with $\beta=1.2$.

The $c_{\text {sep }}$ results for $w>0.5$ agree well with the theory and the experimental results. When $w \leqslant 0.5$, the discrepancy between the model and the theory is, as already discussed, due to the cross-channel tilt of the leading edge of the bore that is not captured in the semigeostrophic solution. The disagreement between the model and 


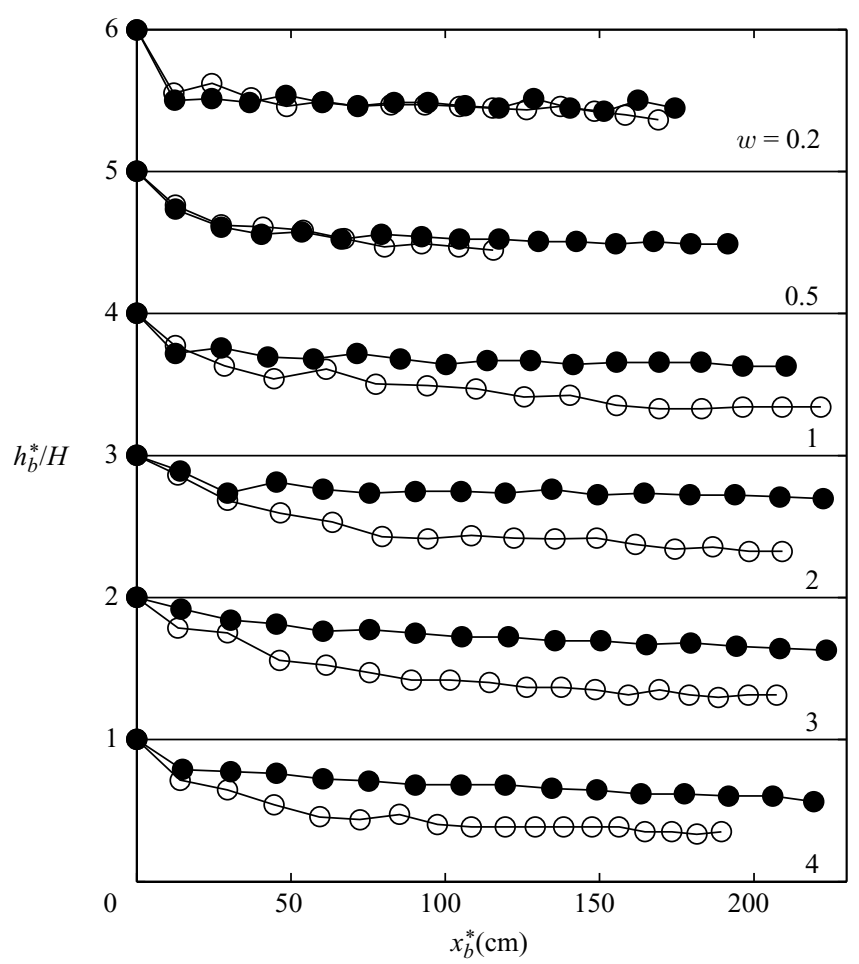

FIGURE 17. Gravity current height $h_{b}^{*} / H$ vs. $x_{b}^{*}$ from a subset of the rectangular domain ROMS model runs with slip walls (solid circles) and no-slip walls (open circles). The non-dimensional channel width $w$ is indicated. At each $w$ the run parameters were identical except for the lateral boundary condition. Each pair of runs is offset by 1 unit on the vertical axis.

the experiments is due to the annular geometry of the experiments. For small $w$ the ratio of the deformation radius to the inner radius of the tank is $\geqslant 1$. The annular domain model with no-slip conditions gives $c_{\text {sep }}=0.42$ and 0.39 for $w=0.25$ and 0.5 , respectively, in better agreement with the laboratory results. The difference in $c_{s e p}$ between slip and no-slip conditions is small.

The numerical model results for $w_{b}$ (at $1.5 L_{R}$ behind $x_{b}$ ) fall below the semigeostrophic theory for $w>0.5$, but are consistent with the new laboratory results. The widths are insensitive to the lateral boundary condition.

The bore height $h_{b}$ is sensitive to the lateral boundary condition. Figure 17 shows $h_{b}$ as a function $x_{b}^{*}$ for a subset of the rectangular domain runs with $H=4 \mathrm{~cm}$ and $g^{\prime}=6.24 \mathrm{~cm} \mathrm{~s}^{-1}$. The bore height was defined as the depth of the $\left(\rho-\rho_{1}\right) / \Delta \rho=0.75$ density contour on the right-hand wall immediately behind the leading edge of the bore. With slip walls, $h_{b}$ initially drops rapidly and then is approximately constant. The no-slip conditions result in a gradual decrease in $h_{b}$ with time. Both conditions are consistent with the local quasi-steady gravity current speed scaling $c_{b}(t)=\beta h_{b}(t)^{1 / 2}$. The slip runs had $\beta=1.16 \pm 0.04$ and $\beta=1.26 \pm 0.17$ for the no-slip runs. The $h_{b}$ at $x=100 \mathrm{~cm}$ from the dam are plotted in (figure $15 \mathrm{c}$ ) for comparison with the semigeostrophic theory. The slip condition results agree quite well with the theory. The no-slip condition results fall within the experimental results. This again implies that the differences between the semigeostrophic theory and the experiments are due primarily to the no-slip boundary condition. 


\section{Lateral viscous drag model}

The numerical model results point to lateral boundary friction, and not Taylor column formation and inertial wave radiation, as the cause of the slow decay in bore speed. A simple model for this process can be derived by considering the loss of momentum owing to friction at a no-slip vertical boundary. It is assumed that the primary momentum balance in the gravity current is between inertia and the pressure gradient provided by buoyancy. Loss of inertia to friction occurs only on a slow time scale. By integrating the momentum equation across the cross-sectional area of the bore, the slow loss of momentum is given by (in dimensional variables, dropping the superscript asterisk for the moment)

$$
A_{b} \frac{\mathrm{d} u}{\mathrm{~d} t} \sim-h_{b} \frac{\tau_{w a l l}}{\rho_{1}} \approx-\frac{v h_{b}}{\delta} u,
$$

where $u$ is the average value of the along channel velocity, $A_{b} \approx w_{b} h_{b} / 2$ is the crosschannel area of the gravity current, $\tau_{\text {wall }} \approx \rho_{1} v u / \delta$ is the stress at the wall, $v$ is the kinematic viscosity, and $\delta=2(v t)^{1 / 2}$ is the boundary-layer thickness. Provided $\delta / w_{b}<1$, the gravity current is essentially inertial. The change to a buoyancy-viscous balance occurs at $t_{c} \sim w_{b}^{2} / v$ (Didden \& Maxworthy 1982; Huppert 1982). The narrowest boundary current in these experiments was $w_{b} \approx 1.5 \mathrm{~cm}$ for $w=4.02$, giving $t_{c} \approx 225 \mathrm{~s}$ with $v=0.01 \mathrm{~cm} \mathrm{~s}^{-1}$. This time is greater than the $85 \mathrm{~s}$ duration of the experiment.

It is possible that the boundary layer will be limited by rotation to the length scale $\delta_{f}=\left(\delta_{E} h_{b}\right)^{1 / 2}$, where $\delta_{E}=(2 v / f)^{1 / 2}$ is the Ekman-layer scale. The time scale for the arrest is the interfacial Ekman-layer spin-down time $t_{E}=4 h_{b} / \delta_{E} f$. However, for this arrest to occur, the vorticity diffused in from the sidewall must be consumed in the interfacial Ekman layer which is present only when $\delta_{E}$ is greater than the interface thickness. This situation is probably not achieved in the experiments or found in the numerical results, though flow characteristic of a weak interfacial Ekman layer is present in the numerical runs. In the worst case for the run at $w=4.02$, the arrest would occur at $t>t_{E}=76 \mathrm{~s}$, near the end of the $85 \mathrm{~s}$ duration of the experiment. It is assumed that interfacial Ekman layers, if present, are weak and do not contribute significantly to the drag or arrest the growth of the sidewall boundary layer. Furthermore, the lack of speed decay in the slip boundary condition ROMS runs indicates that interfacial Ekman drag is weak. It is possible to include both the sidewall boundary-layer arrest and interfacial Ekman drag in the analysis, but it is not necessary.

Substituting $\delta=2(v t)^{1 / 2}$ into (6.1), along with $w_{b} \approx L_{R} / 2$ from the experiments, and non-dimensionalizing $t$ with $f^{-1}$ and $u$ with $\left(g^{\prime} H\right)^{1 / 2}$, gives

$$
\frac{\mathrm{d} u}{\mathrm{~d} t} \sim-2 R e^{-1 / 2} t^{-1 / 2} u,
$$

where $R e=\left(g^{\prime} H\right)^{1 / 2} L_{R} / \nu$. Integration of (6.2) gives

$$
u=U_{0} \mathrm{e}^{-\gamma t^{1 / 2}},
$$

where $\gamma=a R e^{-1 / 2}$. A factor of 4 has been absorbed into the coefficient $a$, which is treated as an adjustable parameter. The parameter $U_{0}$ is the velocity at $t=0$. Since the bore speed $c_{b}=\mathrm{d} x_{b} / \mathrm{d} t \approx u$ from continuity considerations, integration of (6.3) gives

$$
x_{b}=\frac{2 U_{0}}{\gamma^{2}}\left[1-\left(1+\gamma t^{1 / 2}\right) \exp \left(-\gamma t^{1 / 2}\right)\right] \text {. }
$$




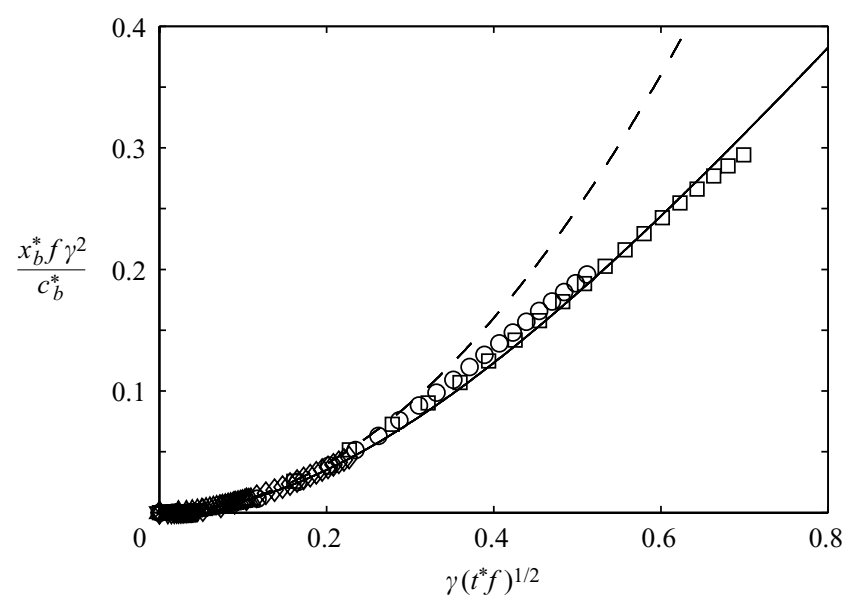

FIGURE 18. The experimental scaled position of the gravity current head $x_{b}^{*} \gamma^{2} f / c_{b}^{*} v s \cdot \gamma\left(t^{*} f\right)^{1 / 2}$. The solid line is from the viscous wall drag theory (6.4) and the dashed line is the constant velocity trajectory $x_{b}^{*}=c_{b}^{*} t^{*}$. The experiments are plotted with $a=2.8$ and $c_{b}^{*}$ from table 1 . The symbols indicate $w=0.25(*), 0.50(\nabla), 0.99(\triangle), 1.95(\diamond), 3.11(\bigcirc), 4.02(\square)$.

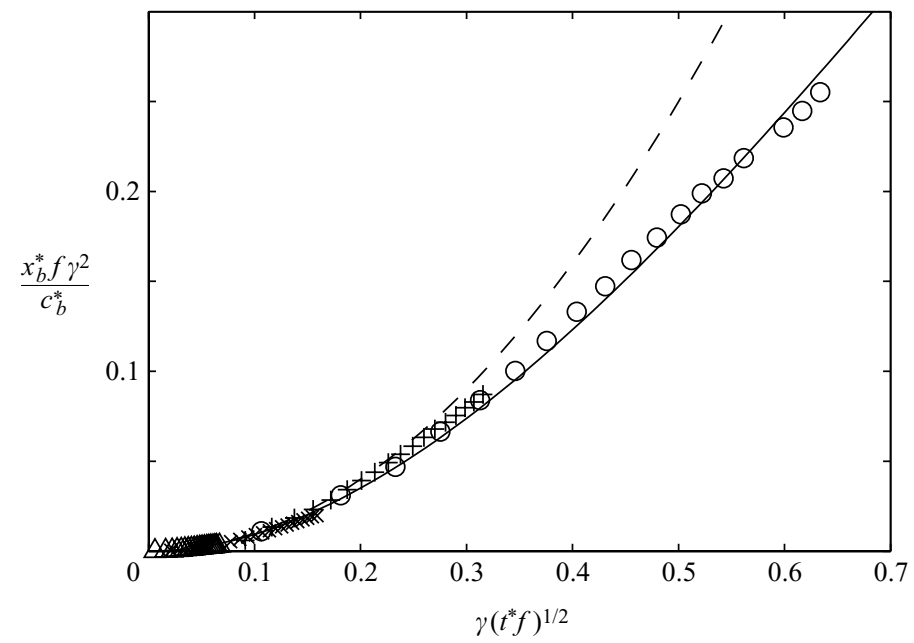

Figure 19. The same as figure 18 except the experimental data are from figure 4 in Griffiths \& Hopfinger (1983) and $a=2$. The symbols $(\bigcirc, \square, \times,+)$ correspond, respectively, to experiments with $g^{\prime}=(0.98,6.7,5.8,0.98) \mathrm{cm} \mathrm{s}^{-2}, H=(9.8,3.8,10,8) \mathrm{cm}$, and $f=(1.03,0.222,1.01,0.517) \mathrm{s}^{-1}$ in a rectangular channel with $w^{*}=30 \mathrm{~cm}$ and $H_{T} \approx 50 \mathrm{~cm}$.

The viscous decay solution (6.4) is used in figure 18 to replot the experimental data in figure 13. The initial gravity current speed $c_{b}$ obtained for $5 H / L_{R}<t<12$ is used for $U_{0}$. The experimental data is fit best (least squares) with $a=2.8$. Analysis of the data from the ROMS runs in the annular channel gives a similarly good agreement with the decay theory with $a=2.4$. The solution (6.4) with $U_{0}=1$ and $a=2.8$ is also shown with experimental and numerical bore trajectories in figure 13.

As a further test of the viscous decay model, the bore trajectory data from the four experiments in figure 4 of GH are reproduced in figure 19 using the viscous scaling. Those experiments had $w=1.3-10$. Best agreement with the model occurs for $a \approx 2$. For three of the experiments $E<3 \times 10^{-4}$, well into the range where inertial wave 
and Taylor column formation drag was proposed as the decay mechanism. The new experiments have $4 \times 10^{-4}<E<2 \times 10^{-3}$. Thus both sets together span the inertial wave radiation and Ekman regimes proposed by GH.

The viscous drag model (6.4) does a good job of collapsing the two experimental data sets and the model runs. It gives a dynamically consistent explanation of the speed decay and predicts correctly those experiments which do not exhibit much decay. The time scale for the decay $\hat{\tau}=\gamma^{-2}=a^{-2} R e$. A reduction of bore speed to $67 \%$ of the initial speed requires an experimental duration of $t \approx 0.16 \hat{\tau}$. These and the earlier experiments were conducted by fixing the channel width and changing $L_{R}$ to vary $w$. Thus, experiments with smaller $L_{R}$ (larger $w$ ), hence smaller $R e$, exhibited the largest speed decay at similar dimensional times.

\section{Summary}

A semigeostrophic theory for the generation of buoyant gravity currents over a deep and quiescent lower layer in a rotating channel has been developed. The theory combines the ageostrophic dynamics of the gravity current head represented by a (local) relation for the gravity current speed with the characteristic solution to the dam-break problem in a uniform width channel. The theory gives relations between the gravity current properties $h_{b}, c_{b}$ and $w_{b}$ and the initial reservoir properties. The crucial parameter is the non-dimensional reservoir width $w$ (scaled by the deformation radius based on the reservoir depth $\left.L_{R}=\left(g^{\prime} H\right)^{1 / 2} / f\right)$. The flow downstream of the dam consists of a rarefaction joined to a uniform (in the along-channel direction) gravity current. The gravity current properties depend upon the bore speed relation used in the analysis, but the qualitative behaviour is independent of the relation. In general, if $w \gtrsim 0.5$, the gravity current will be separated from the left-hand wall, whereas for narrower channels, the gravity current remains attached to both walls. Once detached, the gravity current speed, width and height increase very slowly with $w$.

The theory agrees well with solutions from a single-layer shallow-water model that internally develops a leading bore. The most significant differences between the model and the theory are in the flow near the gravity current head and in the appearance of an internal shock in the gravity current for $w \leqslant 1$. The shock arises from the absence of potential vorticity conservation on fluid parcels flowing through an ageostrophic boundary layer at the bore face. While the non-conservation is here purely an artefact of the numerical solution, it is representative of dissipative processes that would occur in a real turbulent stratified fluid. The model solution shows that the strong offshore jet is produced by fluid flowing toward the head with speeds $>c_{b}$. The jet results in 'detrainment' from the head in qualitative agreement with some of the earlier laboratory experiments.

The new laboratory experiments confirmed the theoretical predictions of the lefthand wall separation point speed $c_{\text {sep }}$ and the general behaviour for $w \leqslant 0.5$, neither of which had been previously examined. However, the experiments showed substantial differences with the theory for $w \gtrsim 1$. The current width and bore height were less than predicted and the bore speed decayed with time. Through numerical solutions to the full three-dimensional hydrostatic equations, these differences were shown to be largely due to the effects of friction. In particular, the no-slip wall boundary condition causes the slow speed decay and a corresponding slow decay in bore height. The tapering of the gravity current width towards the head appears also to be the consequence of internal lateral friction. 
A simple model for the effects of the no-slip wall was developed and shown to agree well with the experiments, including some reported in $\mathrm{GH}$, and the numerical results. The speed decay had previously been attributed by GH to Taylor column formation and inertial wave radiation into the lower layer from the turbulent bore head. The net effect is a drag on the current. However, the success of the viscous decay model over a range of Ekman numbers in their suggested viscous and inertial decay ranges demonstrates that the observed decay is primarily due to viscous boundary effects. This is important since the inertial mechanism, if it were the correct interpretation, could potentially operate at geophysical scales, while the viscous mechanism would probably be insignificant.

An obvious shortcoming of the theoretical model is the restriction to a single active layer. In the non-rotating limit the role of an active lower layer does not significantly affect the gravity current speed when $h_{b} / H_{T}<0.1$ (Benjamin 1968; Klemp et al. 1997; Huppert \& Simpson 1980). With rotation, the effect of the lower layer is similar (SWH; Hacker \& Linden 2002; Martin \& Lane-Serff 2005; Martin, Smeed \& LaneSerff 2005). However, there are difficulties in extending the initial value dam-break solution to two active layers with rotation. The first is that it would be necessary to solve for the evolution of a potential vorticity front in the lower layer along with the gravity current. This front separates lower-layer fluid initially beneath the dammed upper-layer fluid $\left(q=f /\left(H_{T}-H\right)\right)$ from fluid downstream of the dam $\left(q=f / H_{T}\right)$. The second complication is that gravity current heads can generally expected to be dissipative. There is debate about in which layer (if not both) the dissipation occurs (cf. Klemp et al. 1997); however, unless the energy loss is uniform for all streamlines, the potential vorticity is not conserved (Pratt 1983; Martin \& Lane-Serff 2005). The uniform potential vorticity hydraulic theory is not applicable. If the dissipation is assumed to be uniformly distributed within a layer, then the methods developed here could, in principal, be extended to two active layers.

This work was supported by NSF Grants OCE-0095059 and OCE-0132903 and partially completed during the 2003 Geophysical Fluid Dynamics Program at the Woods Hole Oceanographic Institution while J.C. M. was a summer fellow. This is Woods Hole Oceanographic Institution Contribution number 11095.

\section{REFERENCES}

Аввотт, М. B. 1961 On the spreading of one fluid over another. Part ii. The wave front. La Houille Blanche 6, 827-846.

Beardsley, R. C., Dorman, C. E., Rosenfeld, A. \& Winant, C. D. 1987 Local atmospheric forcing during the coastal ocean dynamics experiment 1 . A description of the marine boundary layer and atmospheric conditions over a northern California upwelling region. J. Geophys. Res. 92 , $1467-1488$.

Benjamin, T. B. 1968 Gravity currents and related phenomena. J. Fluid Mech. 31, 209-248.

DidDEN, N. \& MAXWORTHY, T. 1982 Viscous spreading of plane and axisymmetric gravity currents. J. Fluid Mech. 121, 27-42.

Dorman, C. E. 1987 Possible role of gravity currents in northern California's coastal summer wind reversals. J. Geophys. Res. 92, 1497-1506.

Fedorov, A. V. \& Melville, W. K. 1996 Hydraulic jumps at boundaries in rotating fluids. J. Fluid Mech. 324, 55-82.

GARvine, R. W. 1981 Frontal jump conditions for models of shallow, buoyant surface layer hydrodynamics. Tellus 33, 301-312.

Garvine, R. W. 1987 Estuary plumes and fronts in shelf waters: a layer model. J. Phys. Oceanogr. 17, 1897-1896. 
GiLl, A. E. 1977 The hydraulics of rotating-channel flow. J. Fluid Mech. 80, 641-671.

Griffiths, R. W. 1986 Gravity currents in rotating systems. Annu. Rev. Fluid Mech. 18, 59-86.

Griffiths, R. W. \& Hopfinger, E. J. 1983 Gravity currents moving along a lateral boundary in a rotating fluid. J. Fluid Mech. 134, 357-399.

HACKER, J. N. \& Linden, P. F. 2002 Gravity currents in rotating channels. Part 1. Steady-state theory. J. Fluid Mech. 457, 295-324.

Helfrich, K. R., Kuo, A. C. \& Pratt, L. J. 1999 Nonlinear Rossby adjustment in a channel. J. Fluid Mech. 390, 187-222.

HUPPERT, H. 1982 The propagation of two-dimensional and axisymmetric viscous gravity currents over a rigid horizontal surface. J. Fluid Mech. 121, 43-58.

Huppert, H. \& Simpson, J. E. 1980 The slumping of gravity currents. J. Fluid Mech. 99, 785-799.

Klemp, J. B., Rotunno, R. \& Skamrock, W. K. 1994 On the dynamics of gravity currents in a channel. J. Fluid Mech. 269, 169-198.

Klemp, J. B., Rotunno, R. \& Skamrock, W. K. 1997 On the propagation of internal bores. J. Fluid Mech. 331, 81-106.

Kubokawa, A. \& Hanawa, K. 1984 A theory of semigeostrophic gravity waves and its application to the intrusion of a density current along a coast. Part 2 . Intrusion of a density current along a coast of a rotating fluid. J. Oceanogr. Soc. Japan 40, 260-270.

Leveque, R. J. 2002 Finite Volume Methods for Hyperbolic Problems. Cambridge University Press.

Martin, J. R. \& Lane-SerfF, G. F. 2005 Rotating gravity currents. Part 1. Energy loss theory. J. Fluid Mech. 522, 35-62.

Martin, J. R., Smeed, D. A. \& Lane-SerfF, G. F. 2005 Rotating gravity currents. Part 2. Potential vorticity theory. J. Fluid Mech. 522, 63-89.

Munchow, A. \& Garvine, R. W. 1993 Dynamical properties of a buoyancy-driven coastal current. J. Geophys. Res. 98, 20063-20077.

NoF, D. 1987 Penetrating outflows and the dam-break problem. J. Mar. Res. 45, 557-577.

O’Donnell, J. \& Garvine, R. W. 1983 A time dependent, two-layer frontal model of buoyant plume dynamics. Tellus 35A, 73-80.

Pratt, L. J. 1983 On inertial flow over topography. Part 1. Semigeostrophic adjustment to an obstacle. J. Fluid Mech. 131, 195-218.

Pratt, L. J., Helfrich, K. R. \& Chassignet, E. P. 2000 Hydraulic adjustment to an obstacle in a rotating channel. J. Fluid Mech. 404, 117-149.

RENNIE, S., LARGIER, J. L. \& LENTZ, S. J. 1999 Observations of low-salinity coastal pulses downstream of chesapeake bay. J. Geophys. Res. 104, 18 227-18 240.

Schër, C. \& Sмiтh, R. B. 1993 Shallow-water flow past isolated topography. Part i: Vorticity production and wake formation. J. Atmos. Sci. 50, 1373-1400.

ShchepetKin, A. F. \& McWilliams, J. C. 2003 The regional ocean modeling system (ROMS): A split-explicit, free-surface, topography-following coordinates ocean model. Ocean Modeling 9, 347-404.

Simpson, J. E. 1997 Gravity Currents in the Environment and the Laboratory, 2nd edn. Cambridge University Press.

Stern, M. E. \& Helfrich, K. R. 2002 Propagation of a finite-amplitude potential vorticity front along the wall of a stratified fluid. J. Fluid Mech. 468, 179-204.

Stern, M. E., Whitehead, J. A. \& HuA, B. L. 1982 The intrusion of a density current along the coast of a rotating fluid. J. Fluid Mech. 123, 237-265.

StokeR, J. J. 1957 Water Waves. Interscience.

Whitham, G. B. 1974 Linear and Nonlinear Waves. Wiley. 